\title{
UPPERS TO ZERO IN POLYNOMIAL RINGS AND PRÜFER-LIKE DOMAINS
}

\author{
GYU WHAN CHANG \\ DEPARTMENT OF MATHEMATICS \\ UNIVERSITY OF INCHEON, KOREA
}

\author{
MARCO FONTANA \\ DIPARTIMENTO DI MATEMATICA \\ UNIVERSITÀ "ROMA TRE", ITALY
}

\begin{abstract}
Let $D$ be an integral domain and $X$ an indeterminate over $D$. It is well known that (a) $D$ is quasi-Prüfer (i.e, its integral closure is a Prüfer domain) if and only if each upper to zero $Q$ in $D[X]$ contains a polynomial $g \in D[X]$ with content $\boldsymbol{c}_{D}(g)=D$; (b) an upper to zero $Q$ in $D[X]$ is a maximal $t$-ideal if and only if $Q$ contains a nonzero polynomial $g \in D[X]$ with $\boldsymbol{c}_{D}(g)^{v}=D$. Using these facts, the notions of UMt-domain (i.e., an integral domain such that each upper to zero is a maximal $t$-ideal) and quasiPrüfer domain can be naturally extended to the semistar operation setting and studied in a unified frame. In this paper, given a semistar operation $\star$ in the sense of Okabe-Matsuda, we introduce the $\star$-quasi-Prüfer domains. We give several characterizations of these domains and we investigate their relations with the UMt-domains and the Prüfer $v$-multiplication domains.
\end{abstract}

\section{INTRODUCTION AND BACKGROUND RESULTS}

Gilmer and Hoffmann characterized Prüfer domains as those integrally closed domains $D$, such that the extension of $D$ inside its quotient field is a primitive extension 28, Theorem 2]. (Relevant definitions and results are reviewed in the sequel.) Primitive extensions are strictly related with relevant properties of the prime spectrum of the polynomial ring. In particular, from the previous characterization it follows that a Prüfer domain is an integrally closed quasi-Prüfer domain (i.e., an integral domain such that each prime ideal of the polynomial ring contained in an extended prime is extended [5]) [19, Section 6.5]. A quasi-Prüfer domain $D$ can be characterized by the fact that each upper to zero $Q$ in $D[X]$ contains a polynomial $g \in D[X]$ with content $\boldsymbol{c}_{D}(g)=D$ (Theorem [1.1). On the other hand, a "weaker" version of the last property can be used for characterizing upper to zero that are maximal $t$-ideals in the polynomial ring. Recall that $D$ is called a UMt-domain (UM $t$ means "upper to zero is a maximal $t$-ideal") if every upper to zero in $D[X]$ is a maximal $t$-ideal [42, Section 3] and this happens if and only if each upper to zero in $D[X]$ contains a nonzero polynomial $g \in D[X]$ with $\boldsymbol{c}_{D}(g)^{v}=D$ [21, Theorem 1.1]. Using the previous observations, the notions of UMt-domain and quasi-Prüfer domain can be naturally extended to the semistar operation setting and studied in a unified frame. More precisely, given a semistar operation $\star$ in the sense of Okabe-Matsuda [52], we introduce in a natural way the $\star$-quasi-Prüfer domains

Key words and phrases. Prüfer domain, quasi-Prüfer domain, Prüfer $v$-multiplication domain, $\mathrm{UM} t$-domain, star and semistar operation, upper to zero.

2000 Mathematics Subject Classification. 13F05, 13A15, 13G05, $13 \mathrm{~B} 25$.

Email. G.W.C.: whan@incheon.ac.kr; M.F.: fontana@mat.uniroma3.it. 
and semistar analog of other relevant notions like primitive extension and incomparability (INC) property . We give several characterizations of the $\star$-quasi-Prüfer domains and we investigate their relations with the UMt-domains and the Prüfer $v$-multiplication domains [30].

More precisely, let $\star$ be a semistar operation on an integral domain $D$ with quotient field $K$. Among other things, we prove that $D$ is a $\star_{f}$-quasi-Prüfer domain if and only if $D \subseteq K$ is a $\star_{f}$-primitive extension, if and only if $D$ is a $\star_{f}$-INC-domain, if and only if each overring $R$ of $D$ is a $\left(\star_{f}\right)_{\iota}$-quasi-Prüfer domain, where $\iota: D \hookrightarrow R$ is the canonical embedding, if and only if every prime ideal of $\mathrm{Na}\left(D, \star_{f}\right)$ is extended from $D$, if and only if $\mathrm{Na}\left(D, \star_{f}\right)$ is a quasi-Prüfer domain, if and only if the integral closure of $\mathrm{Na}\left(D, \star_{f}\right)$ is a Prüfer domain, if and only if $D_{P}$ is a quasi-Prüfer domain, for each quasi- $\star_{f}$-maximal ideal $P$ of $D$. Moreover, we show that if $\star$ is a (semi)star operation, then $D$ is a $\star_{f}$-quasi-Prüfer domain if and only if $D$ is a $t$-quasi-Prüfer domain and each $\star_{f}$-maximal ideal of $D$ is a $t$-ideal (equivalently, $\widetilde{\star}_{f}=w$ ).

We also show that this general approach sheds new light on some delicate aspects of the classical theories. In particular, we give a contribution to the open problem of whether the integral closure of a UMt-domain is a $\mathrm{P} v \mathrm{MD}$ by showing that $D$ is a UM $t$-domain if and only if the $w$-closure $\widetilde{D}$ of $D$ is a P $v$ MD and the $w$-operations on $D$ and $\widetilde{D}$ are related by $\left(w_{D}\right)_{\tilde{\iota}}=w_{\widetilde{D}}$, where $\widetilde{\iota}: D \hookrightarrow \widetilde{D}$ is the canonical embedding. Moreover, among other results, we provide a positive answer to a Zafrullah's conjecture on the local-global behaviour of the UM $t$ domains 62, page $452]$.

$$
* * * * *
$$

Let $D$ be an integral domain with quotient field $K$. Let $\overline{\boldsymbol{F}}(D)$ denote the set of all nonzero $D$-submodules of $K$ and let $\boldsymbol{F}(D)$ be the set of all nonzero fractional ideals of $D$, i.e. $E \in \boldsymbol{F}(D)$ if $E \in \overline{\boldsymbol{F}}(D)$ and there exists a nonzero $d \in D$ with $d E \subseteq D$. Let $\boldsymbol{f}(D)$ be the set of all nonzero finitely generated $D$-submodules of $K$. Then, obviously $\boldsymbol{f}(D) \subseteq \boldsymbol{F}(D) \subseteq \overline{\boldsymbol{F}}(D)$.

Following Okabe-Matsuda [52, a semistar operation on $D$ is a map $\star: \overline{\boldsymbol{F}}(D) \rightarrow$ $\overline{\boldsymbol{F}}(D), E \mapsto E^{\star}$, such that, for all $x \in K, x \neq 0$, and for all $E, F \in \overline{\boldsymbol{F}}(D)$, the following properties hold:

$\left(\star_{1}\right)(x E)^{\star}=x E^{\star}$

$\left(\star_{2}\right) E \subseteq F$ implies $E^{\star} \subseteq F^{\star}$;

$\left(\star_{3}\right) E \subseteq E^{\star}$ and $E^{\star \star}:=\left(E^{\star}\right)^{\star}=E^{\star}$.

Recall that, given a semistar operation $\star$ on $D$, for all $E, F \in \bar{F}(D)$, the following basic formulas follow easily from the axioms:

$$
\begin{aligned}
(E F)^{\star} & =\left(E^{\star} F\right)^{\star}=\left(E F^{\star}\right)^{\star}=\left(E^{\star} F^{\star}\right)^{\star} ; \\
(E+F)^{\star} & =\left(E^{\star}+F\right)^{\star}=\left(E+F^{\star}\right)^{\star}=\left(E^{\star}+F^{\star}\right)^{\star} ; \\
(E: F)^{\star} & \subseteq\left(E^{\star}: F^{\star}\right)=\left(E^{\star}: F\right)=\left(E^{\star}: F\right)^{\star}, \text { if }(E: F) \neq 0 ; \\
(E \cap F)^{\star} & \subseteq E^{\star} \cap F^{\star}=\left(E^{\star} \cap F^{\star}\right)^{\star}, \text { if } E \cap F \neq(0) ;
\end{aligned}
$$

cf. for instance [18, Theorem 1.2 and p. 174].

A (semi)star operation is a semistar operation that, restricted to $\boldsymbol{F}(D)$, is a star operation (in the sense of [27, Section 32]). It is easy to see that a semistar operation $\star$ on $D$ is a (semi)star operation if and only if $D^{\star}=D$.

If $\star$ is a semistar operation on $D$, then we can consider a map $\star_{f}: \overline{\boldsymbol{F}}(D) \rightarrow \overline{\boldsymbol{F}}(D)$ defined, for each $E \in \overline{\boldsymbol{F}}(D)$, as follows:

$$
E^{\star}:=\bigcup\left\{F^{\star} \mid F \in \boldsymbol{f}(D) \text { and } F \subseteq E\right\} .
$$


It is easy to see that $\star_{f}$ is a semistar operation on $D$, called the semistar operation of finite type associated to $\star$. Note that, for each $F \in \boldsymbol{f}(D), F^{\star}=F_{f}^{\star_{f}}$. A semistar operation $\star$ is called a semistar operation of finite type if $\star=\star_{f}$. It is easy to see that $\left(\star_{f}\right)_{f}=\star_{f}$ (that is, $\star_{f}$ is of finite type).

If $\star_{1}$ and $\star_{2}$ are two semistar operations on $D$, we say that $\star_{1} \leq \star_{2}$ if $E^{\star_{1}} \subseteq E^{\star_{2}}$, for each $E \in \overline{\boldsymbol{F}}(D)$. This is equivalent to say that $\left(E^{\star_{1}}\right)^{\star_{2}}=E^{\star_{2}}=\left(E^{\star_{2}}\right)^{\star_{1}}$, for each $E \in \overline{\boldsymbol{F}}(D)$. Obviously, for each semistar operation $\star$ defined on $D$, we have $\star_{f} \leq \star$. Let $d_{D}$ (or, simply, $d$ ) be the identity (semi)star operation on $D$, clearly $d \leq \star$, for all semistar operation $\star$ on $D$.

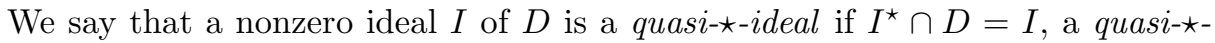
prime if it is a prime quasi- $\star$-ideal, and a quasi- $\star$-maximal if it is maximal in the set of all proper quasi- $\star$-ideals. A quasi- $\star-$ maximal ideal is a prime ideal. It is possible to prove that each proper quasi- $\star_{f}$-ideal is contained in a quasi- $\star_{f}$-maximal ideal. More details can be found in [24, page 4781]. We will denote by $\operatorname{QMax}^{\star}(D)$ (resp., $\operatorname{QSpec}^{\star}(D)$ ) the set of the quasi- $\star$-maximal ideals (resp., quasi- $\star$-prime ideals) of $D$. When $\star$ is a (semi)star operation the notion of quasi- $\star$-ideal coincides with the "classical" notion of $\star$-ideal (i.e., a nonzero ideal $I$ such that $I^{\star}=I$ ).

The $\star$-dimension of $D$, denoted by $\operatorname{dim}^{\star}(D)$, is defined by the supremum of $\left\{n \mid P_{1} \subsetneq P_{2} \subsetneq \cdots \subsetneq P_{n}\right.$ is a chain of quasi- $\star$-prime ideals of $\left.D\right\}$. Thus, when $\star$ is a semistar operation of finite type and $D$ is not a field, $\operatorname{dim}^{\star}(D)=1$ if and only if each quasi- $\star$-maximal ideal of $D$ has height-one.

If $\Delta$ is a set of prime ideals of an integral domain $D$, then the semistar operation $\star_{\Delta}$ defined on $D$ as follows

$$
E^{\star \Delta}:=\bigcap\left\{E D_{P} \mid P \in \Delta\right\}, \text { for each } E \in \bar{F}(D),
$$

is called the spectral semistar operation associated to $\Delta$. A semistar operation $\star$ of an integral domain $D$ is called a spectral semistar operation if there exists a subset $\Delta$ of the prime spectrum of $D, \operatorname{Spec}(D)$, such that $\star=\star \Delta$.

When $\Delta:=\operatorname{QMax}^{\star_{f}}(D)$, we set $\widetilde{\star}:=\star_{\Delta}$, i.e.

$$
E^{\widetilde{*}}:=\bigcap\left\{E D_{P} \mid P \in \operatorname{QMax}^{\star}(D)\right\} \text {, for each } E \in \overline{\boldsymbol{F}}(D) \text {. }
$$

A semistar operation $\star$ is stable if $(E \cap F)^{\star}=E^{\star} \cap F^{\star}$, for each $E, F \in \overline{\boldsymbol{F}}(D)$. Spectral semistar operations are stable [18, Lemma 4.1 (3)]. In particular, $\widetilde{\star}$ is a semistar operation stable and of finite type [18, Corollary 3.9].

By $v_{D}$ (or, simply, by $v$ ) we denote the $v$-(semi)star operation defined as usual by $E^{v}:=(D:(D: E))$, for each $E \in \bar{F}(D)$. By $t_{D}$ (or, simply, by $t$ ) we denote $\left(v_{D}\right)_{f}$ the $t-\left(\right.$ semi)star operation on $D$ and by $w_{D}$ (or just by $w$ ) the stable semistar operation of finite type associated to $v_{D}$ (or, equivalently, to $t_{D}$ ), considered by F.G. Wang and R.L. McCasland in [60] (cf. also [29]); i.e., $w_{D}:=\widetilde{v_{D}}=\widetilde{t_{D}}$. Clearly $w_{D} \leq t_{D} \leq v_{D}$. Moreover, it is easy to see that for each (semi)star operation $\star$ of $D$, we have $\star \leq v_{D}$ and $\star_{f} \leq t_{D}$ (cf. also [27, Theorem 34.1 (4)]).

If $I \in \overline{\boldsymbol{F}}(D)$, we say that $I$ is $\star-$ finite if there exists $J \in \boldsymbol{f}(D)$ such that $J^{\star}=I^{\star}$. It is immediate to see that if $\star_{1} \leq \star_{2}$ are semistar operations and $I$ is $\star_{1}$-finite, then $I$ is $\star_{2}$-finite. In particular, if $I$ is $\star_{f}$-finite, then it is $\star$-finite. The converse is not true and it is possible to prove that $I$ is $\star_{f}$-finite if and only if there exists $J \in \boldsymbol{f}(D), J \subseteq I$, such that $J^{\star}=I^{\star}$ [25, Lemma 2.3].

If $I$ is a nonzero ideal of $D$, we say that $I$ is $\star$-invertible if $\left(I I^{-1}\right)^{\star}=D^{\star}$. From the definitions and from the fact that $\operatorname{QMax}^{\star_{f}}(D)=\operatorname{QMax}^{\widetilde{\star}}(D)$ 24, Corollary 
$3.5(2)]$ it follows easily that an ideal $I$ is $\tilde{\star}$-invertible if and only if $I$ is $\star_{f}$-invertible. If $I$ is $\star_{f}$-invertible, then $I$ and $I^{-1}$ are $\star_{f}$-finite [25, Proposition 2.6].

Let $R$ be an overring of an integral domain $D$, let $\iota: D \hookrightarrow R$ be the canonical embedding and let $\star$ be a semistar operation of $D$. We denote by $\star_{\iota}$ the semistar operation of $R$ defined by $E^{\star_{\iota}}:=E^{\star}$, for each $E \in \overline{\boldsymbol{F}}(R)(\subseteq \overline{\boldsymbol{F}}(D))$. Let $*$ be a semistar operation of $R$ and let $*^{\iota}$ be the semistar operation on $D$ defined by $E^{*^{\iota}}:=(E R)^{*}$, for each $E \in \overline{\boldsymbol{F}}(D)$. It is not difficult to see that $\left(*^{\iota}\right)_{f}=\left(*_{f}\right)^{\iota}$ and if $\star$ is a semistar operation of finite type (resp., a stable semistar operation) of $D$ then $\star_{\iota}$ is a semistar operation of finite type (resp., a stable semistar operation) of $R$ (cf. for instance [23, Proposition 2.8] and [55, Propositions 2.11 and 2.13]).

\section{QUASI-PRÜFER DOMAINS}

Let $D$ be an integral domain with quotient field $K$, and let $\boldsymbol{X}$ be a nonempty set of indeterminates over $K$. For each polynomial $f \in K[\boldsymbol{X}]$, we denote by $\boldsymbol{c}_{D}(f)$ (or, simply, $\boldsymbol{c}(f)$ ) the content on $D$ of the polynomial $f$, i.e., the (fractional) ideal of $D$ generated by the coefficients of $f$. For each fractional ideal $J$ of $D[\boldsymbol{X}]$, with $J \subseteq$ $K[\boldsymbol{X}]$, we denote by $\boldsymbol{c}_{D}(J)$ (or, simply, $\left.\boldsymbol{c}(J)\right)$ the (fractional) ideal $\left\{\boldsymbol{c}_{D}(f) \mid f \in J\right\}$ of $D$. Obviously, for each ideal $J$ in $D[\boldsymbol{X}], J \cap D \subseteq \boldsymbol{c}_{D}(J)$ and $(J \cap D)[\boldsymbol{X}] \subseteq J \subseteq$ $\boldsymbol{c}_{D}(J)[\boldsymbol{X}]$.

Taking the properties of prime ideals in polynomial extensions of Prüfer domains as a starting point, the quasi-Prüfer notion was introduced in [5 for arbitrary rings (not necessarily domains). As in [19, page 212], we say that $D$ is a quasi-Prüfer domain if for each prime ideal $P$ of $D$, if $Q$ is a prime ideal of $D[\boldsymbol{X}]$ with $Q \subseteq P[\boldsymbol{X}]$, then $Q=(Q \cap D)[\boldsymbol{X}]$. It is well known that an integral domain is a Prüfer domain if and only if it is integrally closed and quasi-Prüfer [27, Theorem 19.15].

Consider now the following condition:

$\left(\mathbf{q} \mathbf{P}^{\prime}\right)$ if $Q$ is a prime ideal of $D[\boldsymbol{X}]$ with $\boldsymbol{c}_{D}(Q) \subsetneq D$, then $Q=(Q \cap D)[\boldsymbol{X}]$.

It is clear that $D$ satisfies $\left(\mathbf{q} \mathbf{P}^{\prime}\right)$ if and only if $D$ is a quasi-Prüfer domain. Therefore, an integrally closed domain $D$ is a Prüfer domain if and only if $D$ satisfies $\left(\mathbf{q} \mathbf{P}^{\prime}\right)$.

Let $D \subseteq R$ be an extension of integral domains, and let $P$ be a prime ideal of $D$. We say that $D \subseteq R$ satisfies INC at $P$ if whenever $Q_{1}$ and $Q_{2}$ are prime ideals of $R$ such that $Q_{1} \cap D=P=Q_{2} \cap D$, then $Q_{1}$ and $Q_{2}$ are incomparable. If $D \subseteq R$ satisfies INC at every prime ideal of $D, D \subseteq R$ is said an INC-extension. The domain $D$ is an INC-domain if, for each overring $R$ of $D, D \subseteq R$ is an INCextension.

An element $u \in R$ will be said to be primitive over $D$ if $u$ is a root of a primitive polynomial on $D$ (i.e., a nonzero polynomial $f \in D[X]$ with $\left.\boldsymbol{c}_{D}(f)=D\right)$. The extension $D \subseteq R$ is called a primitive extension (or, a P-extension [28]) if each element of $R$ is primitive over $D$.

A nonzero prime ideal $Q$ in the polynomial ring $D[X]$ is called an upper to zero (McAdam's terminology) if $Q \cap D=(0)$. Let $P:=Q \cap D$, if $Q=P[X]$ then $Q$ is called an extended prime of $D[X]$ (more details can be found in [39]).

Recall that Gilmer and Hoffmann characterized Prüfer domains as those integrally closed domains $D$, such that the embedding of $D$ inside its quotient field is a P-extension [28, Theorem 2], and that D. Dobbs in [10] characterized P-extensions in terms of INC-domains. The natural link between quasi-Prüfer domains and primitive extensions is recalled in the following theorem, where we collect several 
useful characterizations of quasi-Prüfer domains (cf. also the very recent survey paper by E. Houston [39]).

Theorem 1.1. Let $D$ be an integral domain with quotient field $K$, let $X$ be an indeterminate over $D$ and let $\mathcal{N}:=\left\{g \in D[X] \mid \boldsymbol{c}_{D}(g)=D\right\}$ be the set of primitive polynomials over $D$. Then the following statements are equivalent.

(1) $D$ is a quasi-Prüfer domain.

$\left(1^{\prime}\right) D$ satisfies $\left(\mathbf{q} \mathbf{P}^{\prime}\right)$ for one indeterminate.

(2) Each upper to zero in $D[X]$ contains a polynomial $g \in D[X]$ with $\boldsymbol{c}_{D}(g)=$ $D$.

(3) If $Q$ is an upper to zero in $D[X]$, then $\boldsymbol{c}_{D}(Q)=D$.

(4) $D \subseteq K$ is a primitive extension.

(5) $D$ is an INC-domain.

(6) The integral closure of $D$ is a Prüfer domain.

(7) Each overring of $D$ is a quasi-Prüfer domain.

(8) Each prime ideal of $D[X]_{\mathcal{N}}$ is extended from $D$.

(9) $D[X]_{\mathcal{N}}$ is a quasi-Prüfer domain.

(10) The integral closure of $D[X]_{\mathcal{N}}$ is a Prüfer domain.

(11) $D_{M}$ is a quasi-Prüfer domain, for each maximal ideal $M$ of $D$.

Proof. $(1) \Leftrightarrow(4) \Leftrightarrow(5) \Leftrightarrow(6) \Leftrightarrow(7)$ and $(9) \Leftrightarrow(10)$ by [19, Corollary 6.5.14]. Moreover, $(3) \Leftrightarrow(6)$ by [3, Theorem 2.7]

$(2) \Leftrightarrow(3),(1) \Leftrightarrow(11)$ and $(1) \Rightarrow\left(1^{\prime}\right)$ are clear.

$\left(1^{\prime}\right) \Rightarrow(3)$ If $Q$ is an upper to zero, then $Q \neq(Q \cap D)[X]$, and thus, by $\left(1^{\prime}\right)$, $\boldsymbol{c}_{D}(Q)=D$.

$(6) \Leftrightarrow(10)$ Let $\bar{D}$ be the integral closure of $D$, and let $\overline{\mathcal{N}}:=\left\{h \in \bar{D}[X] \mid \boldsymbol{c}_{\bar{D}}(h)=\right.$ $\bar{D}\}$. Then it is clear that $\bar{D}[X]_{\mathcal{N}}=\bar{D}[X]_{\mathcal{N}}$. Moreover, $\bar{D}[X]_{\mathcal{N}}$ coincides with the integral closure of $D[X]_{\mathcal{N}}$ [1, Chapter 5, Proposition 5.12 and Exercise 9]. Finally, recall that $\bar{D}$ is a Prüfer domain if and only if $\bar{D}[X]_{\mathcal{N}}$ is a Prüfer domain [27, Theorem 33.4].

$\left(1^{\prime}\right) \Rightarrow(8)$ Let $\mathfrak{Q}$ be a prime ideal of $D[X]_{\mathcal{N}}$. Then $\mathfrak{Q}=Q D[X]_{\mathcal{N}}$ for some prime ideal $Q$ of $D[X]$. Since $\mathfrak{Q} \subsetneq D[X]_{\mathcal{N}}, Q \cap \mathcal{N}=\emptyset$; hence $\boldsymbol{c}(Q) \subsetneq D$. So, by $\left(1^{\prime}\right)$, $Q=(Q \cap D)[X]$. Thus $\mathfrak{Q}=(Q \cap D) D[X]_{\mathcal{N}}$.

$(8) \Rightarrow\left(1^{\prime}\right)$ Let $Q$ be prime ideal of $D[X]$ such that $\boldsymbol{c}(Q) \subsetneq D$. Then $Q \cap \mathcal{N}=\emptyset$ and thus $Q D[X]_{\mathcal{N}}$ is a prime ideal of $D[X]_{\mathcal{N}}$. Therefore, by $(8),(Q \cap D) D[X]_{\mathcal{N}}=$ $Q D[X]_{\mathcal{N}}$, and hence $Q=(Q \cap D)[X]$.

In view of the extensions to the case of semistar operations, we introduce the following notation. Let $\star$ be a semistar operation on $D$, if $\mathcal{N}^{\star}:=\{g \in D[X] \mid$ $g \neq 0$ and $\left.\boldsymbol{c}_{D}(g)^{\star}=D^{\star}\right\}$, then we set $\mathrm{Na}(D, \star):=D[X]_{\mathcal{N}^{\star}}$. The ring of rational functions $\mathrm{Na}(D, \star)$ is called the $\star-N a g a t a$ domain of $D$. When $\star=d$ the identity (semi)star operation on $D, \mathcal{N}^{d}=\mathcal{N}$ (the multiplicative set of $D[X]$ introduced in Theorem 1.1) and we set simply $\mathrm{Na}(D)$ instead of $\mathrm{Na}(D, d)=D[X]_{\mathcal{N}}$. Note that $\mathrm{Na}(D)$ coincides with the classical Nagata domain $D(X)$ (cf. for instance [53, Chapter I, §6 page 18] and [27, Section 33]).

Recall from [24, Propositions 3.1 and 3.4] that:

(a) $\mathcal{N}^{\star}=\mathcal{N}^{\star_{f}}=\mathcal{N}^{\star}=D[X] \backslash \bigcup\left\{P[X] \mid P \in \operatorname{QMax}^{\star_{f}}(D)\right\}$ is a saturated multiplicatively closed subset of $D[X]$.

(b) $\mathrm{Na}(D, \star)=\mathrm{Na}\left(D, \star_{f}\right)=\mathrm{Na}(D, \widetilde{\star})=\bigcap\left\{D_{P}(X) \mid P \in \operatorname{QMax}^{\star_{f}}(D)\right\}$.

(c) $\operatorname{QMax}^{\star} f(D)=\{M \cap D \mid M \in \operatorname{Max}(\mathrm{Na}(D, \star))\}$. 
(d) $E^{\tilde{\star}}=E \cdot \mathrm{Na}(D, \star) \cap K$, for each $E \in \overline{\boldsymbol{F}}(D)$.

Remark 1.2. (a) It is well known that an upper to zero $Q$ in $D[X]$ is a maximal $t$-ideal if and only if $Q$ contains a nonzero polynomial $g \in D[X]$ with $\boldsymbol{c}_{D}(g)^{t}$ (= $\left.\boldsymbol{c}_{D}(g)^{v}\right)=D$ [42, Theorem 1.4]. Recall that $D$ is called a UMt-domain if every upper to zero in $D[X]$ is a maximal $t$-ideal [42, Section 3]. An overring $R$ of $D$ is called $t$-linked to $D$ if, for each nonzero finitely generated ideal $I$ of $D,(D: I)=D$ implies $(R: I R)=R$ (cf. for instance [11] and [12]). Recall that UMt-domains can be characterized by weaker $(t-)$ versions of some of the statements of Theorem 1.1 since the following statements are equivalent:

$\left(1_{t}\right) \quad D$ is a UMt-domain.

$\left(2_{t}\right)$ Each upper to zero in $D[X]$ contains a nonzero polynomial $g \in D[X]$ with $\boldsymbol{c}_{D}(g)^{t}=\boldsymbol{c}_{D}(g)^{v}=D$.

$\left(3_{t}\right)$ If $Q$ is an upper to zero in $D[X]$, then $\boldsymbol{c}_{D}(Q)^{t}=D$.

$\left(7_{t}\right)$ Each $t$-linked overring to $D$ is a UMt-domain.

$\left(8_{t}\right)$ Each prime ideal of $\mathrm{Na}(D, t)$ is extended from $D$.

$\left(11_{t}\right) D_{P}$ is a quasi-Prüfer domain, for each maximal $t$-ideal $P$ of $D$.

For the proof see [21, Theorem 1.1] and [7, Theorem 2.6 $(1) \Leftrightarrow(8)]$.

(b) Note that if $P \subseteq Q$ are two primes ideals in a UMt-domain with $P \neq(0)$ and if $Q$ is a prime $t$-ideal then $P$ is also a prime $t$-ideal [21, Corollary 1.6].

(c) With the notation introduced just before this remark, one of the arguments in the proof of $(6) \Leftrightarrow(10)$ in Theorem 1.1 shows that, for any integral domain $D$, the integral closure of $\mathrm{Na}(D)$ is $\mathrm{Na}(\bar{D})$.

(d) Recall that an integral domain $D$ is called a Prüfer v-multiplication domain (for short, $P v M D$ ) if each nonzero finitely generated ideal of $D$ is $t$-invertible or, equivalently, if $\left(F F^{-1}\right)^{t}=D$, for each $F \in \boldsymbol{f}(D)$ 30. It is known that a domain $D$ is an integrally closed domain and a UMt-domain if and and only if $D$ is a $\mathrm{P} v \mathrm{MD}$ [42, Proposition 3.2]. But M. Zafrullah [62, page 452] mentioned a problem that seems to be still open: is the integral closure of a UMt-domain a PvMD? We will give some contributions to this problem in the following Corollaries 2.17 and 2.18,

A related question is the following: if the integral closure $\bar{D}$ of an integral domain $D$ is a PvMD what can be said about the UMt-ness of D? An answer to this question was recently given by Chang and Zafrullah [7, Remark 2.7] where they provide an example of a non-UMt domain with the integral closure which is a $\mathrm{P} v \mathrm{MD}$.

Using the notion of UMt-domain (recalled in the previous remark) we have further characterizations of a quasi-Prüfer domain (cf. Theorem 1.1).

Corollary 1.3. The following statements are equivalent for an integral domain D.

(1) $D$ is a quasi-Prüfer domain.

(12) Each overring of $D$ is a UMt-domain.

(13) $D$ is a UMt-domain and each maximal ideal of $D$ is a $t$-ideal.

(14) $D$ is a UMt-domain and $d=w$.

In particular, in a quasi-Prüfer domain every nonzero prime ideal is a $t$-ideal.

Proof. $(1) \Leftrightarrow(12)$ by [21, Corollary 3.11] and Theorem 1.1$]((1) \Leftrightarrow(6))$.

$(1) \Rightarrow(13)$ If $Q$ is an upper to zero in $D[X]$, then $Q$ contains a nonzero polynomial $g \in D[X]$ with $\boldsymbol{c}_{D}(g)=D$ (Theorem $1.1((1) \Rightarrow(2))$ ). Clearly $\boldsymbol{c}_{D}(g)^{t}=D$, and thus $D$ is a UMt-domain (Remark 1.2 (a) or [42, Theorem 1.4]). Let $M$ be a maximal 
ideal of $D$. If $M^{t}=D$, there is a polynomial $0 \neq h \in M[X]$ such that $\boldsymbol{c}_{D}(h)^{t}=D$. It is easy to see that $h D[X]_{M[X]} \cap D=(0)$. In this situation, there exists an upper to zero $Q^{\prime}$ in $D[X]$ such that $h D[X] \subseteq Q^{\prime} \subseteq M[X]$ 9, Lemma 1.1 (b)]. Hence $Q^{\prime}$ (and thus $M[X])$ contains a nonzero polynomial $g^{\prime}$ with $\boldsymbol{c}_{D}\left(g^{\prime}\right)=D$ by Theorem 1.1 $((1) \Rightarrow(2))$, thus $D[X]=\boldsymbol{c}_{D}\left(g^{\prime}\right) D[X] \subseteq M[X]$, a contradiction. Therefore $M^{t} \subsetneq D$, hence $M$ is a $t$-ideal.

$(13) \Rightarrow(1)$ Let $Q$ be an upper to zero in $D[X]$. Since we are assuming that $D$ is a UMt-domain, then $Q$ is a maximal $t$-ideal of $D[X]$, and hence $Q$ contains a polynomial $0 \neq g \in D[X]$ with $\boldsymbol{c}_{D}(g)^{t}=\boldsymbol{c}_{D}(g)^{v}=D$ [42, Theorem 1.4]. Furthermore, by assumption, if $M$ is a maximal ideal of $D$, then $\boldsymbol{c}_{D}(g) \nsubseteq M$ since $M$ is a $t$-ideal. Hence $\boldsymbol{c}_{D}(g)=D$, and thus $D$ is a quasi-Prüfer domain by Theorem $1.1((2) \Rightarrow(1))$.

$(13) \Rightarrow(14)$ Note that from (13) it follows easily that $\operatorname{Max}(D)=\operatorname{Max}^{t}(D)$. Thus $d=\widetilde{d}=\widetilde{t}=w$.

$(14) \Rightarrow(13)$ Under the present assumption $\operatorname{Max}(D)=\operatorname{Max}^{w}(D)$ and it is known that $\operatorname{Max}^{w}(D)=\operatorname{Max}^{t}(D)$ (cf. for instance [24, Corollary $\left.3.5(2)\right]$ ).

The last statement is an easy consequence of the fact that a quasi-Prüfer domain is a UMt-domain and of Remark 1.2 (b).

Remark 1.4. (a) From the previous Corollary $1.3((1) \Leftrightarrow(13))$, we easily deduce that the condition $\left(11_{t}\right)$ in Remak 1.2 (a), that characterizes the UMt-domains, is equivalent to the following:

$\left(11_{t}^{\prime}\right) D_{P}$ is a $U M t_{D_{P}}$-domain and $P D_{P}$ is a maximal $t_{D_{P}}$-ideal of $D_{P}$, for each maximal $t_{D}$-ideal $P$ of $D$.

(Cf. also [21, Theorem 1.5] and [39, Theorem 3.13].) This result provides a positive answer to the following Zafrullah's conjecture [62, page 452]: an integral domain $D$ is a $U M t_{D}$-domain if and only if $D_{M}$ is a $U M t_{D_{M}}$-domain, for each maximal ideal $M$ of $D$, and $D$ is well behaved (i.e., a domain such that prime $t$-ideals of the domain extend to prime $t$-ideals in the rings of fractions of $D$ ).

As a matter of fact, the "only if part", on which was based the conjecture, was already proved in [21, Propositions 1.2 and 1.4]; the "if part" follows from the equivalence of $\left(11_{t}^{\prime}\right)$ with $\left(1_{t}\right)$ of Remark 1.2 (a).

(b) Note that the condition (12) in the previous Corollary 1.3 can be stated in the following equivalent form:

$\left(12^{\prime}\right) D$ is a UMt-domain and each overring of $D$ is t-linked to $D$.

(Cf. [13, Theorem 2.4].)

(c) In relation with (14) of Corollary 1.3, we recall that the domains for which $d=w$ were introduced and studied in [49] under the name of $D W$-domains (cf. also [56] for further information on these domains). A DW-domain $D$ can be characterized by the property that each overring $R$ of $D$ is $t$-linked to $D$ (cf. [13, Theorem 2.6], [12] and [49, Proposition 2.2]).

Corollary 1.5. Let $D$ be a quasi-Prüfer domain. Then $\operatorname{dim}(D)=\operatorname{dim}^{w}(D)=$ $\operatorname{dim}^{t}(D)=\operatorname{dim}(\mathrm{Na}(D))$.

Proof. This follows because, in the present situation, $d=w$, every nonzero prime ideal of $D$ is a $t$-ideal (Corollary 1.3) and each prime ideal of $\mathrm{Na}(D)$ is extended from $D$ by Theorem $1.1((1) \Rightarrow(8))$. 
Remark 1.6. Note that even in the Prüfer domain case, it might happen that $\operatorname{dim}(D)\left(=\operatorname{dim}^{t}(D)\right) \geqslant \operatorname{dim}^{v}(D)$. For instance take a nondiscrete valuation domain. In this case, the maximal ideal is not a $v$-ideal.

\section{2. ^-QUASI-PRÜFER DOMAINS AND UPPERS TO ZERO}

Let $\star$ be a semistar operation on an integral domain $D$. We want to introduce a semistar analog to the notion of quasi-Prüfer domain and to the related notion of $\mathrm{UM} t$-domain.

We say that an integral domain $D$ is a $\star$-quasi-Prüfer domain if the following property holds:

$(\star \mathbf{q P})$ if $Q$ is a prime ideal in $D[X]$ and $Q \subseteq P[X]$, for some $P \in \operatorname{QSppec}^{\star}(D)$, then $Q=(Q \cap D)[X]$.

It is clear from the definition that the $d$-quasi-Prüfer domains are exactly the quasi-Prüfer domains.

Lemma 2.1. Let $\star$ be a semistar operation on an integral domain D. The following statements are equivalent:

(i) $D$ is a $\star$-quasi-Prüfer domain.

(ii) Let $Q$ be an upper to zero in $D[X]$, then $\boldsymbol{c}_{D}(Q) \nsubseteq P$, for each $P \in$ $\operatorname{QSpec}^{\star}(D)$.

(iii) Let $Q$ be an upper to zero in $D[X]$, then $Q \nsubseteq P P[X]$, for each $P \in \operatorname{QSpec}^{\star}(D)$.

(iv) $D_{P}$ is a quasi-Prüfer domain, for each $P \in \operatorname{QSpec}^{\star}(D)$.

Proof. (i) $\Rightarrow$ (iii) follows immediately from the definition.

(iii) $\Rightarrow$ (ii) If $Q$ is an upper to zero then by assumption $Q \nsubseteq \nsubseteq P[X]$, for all $P \in$ $\operatorname{QSpec}^{\star}(D)$. Then $\boldsymbol{c}(Q) \nsubseteq \subseteq P$, for each $P \in \mathrm{QSpec}^{\star}(D)$, since $Q \subseteq \boldsymbol{c}_{D}(Q)[X]$.

(ii) $\Rightarrow$ (i) Assume that $Q$ is a prime ideal in $D[X]$ such that $(Q \cap D)[X] \subsetneq Q \subseteq$ $P[X]$, for some $P \in \operatorname{QMax}^{\star}(D)$. Then we can find an upper to zero $Q_{1}$ in $D[X]$ such that $Q_{1} \subseteq Q\left[9\right.$, Theorem A]. Thus $\boldsymbol{c}_{D}\left(Q_{1}\right) \subseteq \boldsymbol{c}_{D}(Q) \subseteq P$, for some $P \in$ $\operatorname{QSpec}^{\star}(D)$, and this contradicts the present hypothesis.

(i) $\Rightarrow$ (iv) Let $P \in \operatorname{QSpec}^{\star}(D)$. In order to show that $D_{P}$ is a quasi-Prüfer domain, we prove the condition $\left(1^{\prime}\right)$ of Theorem 1.1. If $Q$ is a prime ideal of $D_{P}[X]$ with $\boldsymbol{c}_{D_{P}}(Q) \subsetneq D_{P}$, then $\boldsymbol{c}_{D_{P}}(Q) \subseteq P D_{P}$, and hence $Q \subseteq P D_{P}[X]$. So $Q \cap D[X] \subseteq$ $P[X]$, and by (i) we have $Q \cap D[X]=(Q \cap D)[X]$. Hence $Q=\left(Q \cap D_{P}\right)[X]$. Thus $D_{P}$ is a quasi-Prüfer domain.

(iv) $\Rightarrow$ (i) Let $Q$ be a prime ideal of $D[X]$ such that $Q \subseteq P[X]$ for some $P \in$ $\operatorname{QSpec}^{\star}(D)$. Then $Q D_{P}[X] \subseteq P D_{P}[X]$, and hence $Q D_{P}[X]=\left(Q D_{P}[X] \cap D_{P}\right)[X]$ by (iv). Thus $Q=\left(Q D_{P}[X] \cap D_{P}\right)[X] \cap D[X]=(Q \cap D)[X]$.

Since a quasi- $\star$-ideal is also a quasi- $\star_{f}$-ideal, it is clear that $\star_{f}$-quasi-Prüfer implies $\star$-quasi-Prüfer. Recall that every quasi- $\star_{f}$-ideal is contained in a quasi- $\star_{f}$-maximal ideal and each quasi- $\star_{f}$-maximal ideal is a prime ideal [24, Lemma 2.3]. Therefore, the set $\operatorname{QSpec}^{\star} f(D)$ is always nonempty. On the other hand $\operatorname{QSpec}^{\star}(D)$ can be empty and in this case the notion of $\star$-quasi-Prüfer domain can be very weak.

Note also that, when $\star$ is a semistar operation of finite type, in the condition $(\star \mathbf{q P})$ and in the properties (ii), (iii), and (iv) of the previous Lemma 2.1 we can replace $\operatorname{QSpec}^{\star}(D)$ with $\operatorname{QMax}^{\star}(D)$, obtaining equivalent statements.

Example 2.2. Example of a $\star$-quasi-Prüfer domain which is not a $\star_{f}$-quasi-Prüfer domain. 
Let $W$ be a 1-dimensional nondiscrete valuation domain with maximal ideal $N$ and residue field $k:=W / N$. Let $Z$ be an indeterminate over $W$. Passing to the Nagata's ring $V:=W(Z)$, it is wellknown that $V$ is also a 1-dimensional nondiscrete valuation domain, with maximal ideal $M:=N(Z)$ and residue field $k(Z)$ (cf. [27, Theorem 33.4] and 44, Theorem 14.1 and Corollary 15.2]). Let $\pi: V \rightarrow V / N=k(Z)$ be the canonical projection and let $D=\pi^{-1}(k)$. Clearly, $D$ is an integrally closed 1-dimensional pseudo-valuation domain with maximal ideal $M$ and with associated valuation overring $V=(M: M)$ [33, Theorem 2.10]. Note that $V$ has no divisorial primes, since $M$ is not finitely generated 27, Exercise 12 , page 431] and that the $t$-operation on a valuation domain coincides with $d$ the identity (semi)star operation. Let $\iota: D \hookrightarrow V$ be the canonical embedding and let $\star:=\left(v_{V}\right)^{\iota}$ be the semistar operation on $D$ defined by $E^{\star}:=(E V)^{v_{V}}$, for each $E \in \overline{\boldsymbol{F}}(D)$. Note that $\star$ is not of finite type and more precisely it is not difficult to see that:

$$
\star_{f}=\left(\left(v_{V}\right)^{\iota}\right)_{f}=\left(\left(v_{V}\right)_{f}\right)^{\iota}=\left(t_{V}\right)^{\iota}=\left(d_{V}\right)^{\iota} \quad \text { [55, Proposition 2.13]. }
$$

Therefore $E^{\star_{f}}=E V$, for each $E \in \overline{\boldsymbol{F}}(D)$. In particular, $M$ is a (quasi-) $\star_{f}$-maximal ideal of $D$. Note that $D$ is not a $\star_{f}$-quasi-Prüfer domain since, if $X$ is an indeterminate over $D, \operatorname{dim}(D[X])=3$, because there exists an upper to zero $Q$ in $D[X]$ contained in $M[X]$ [34, Theorem 2.5 and Remark 2.6]. On the other hand $D$ is trivially a $\star$-quasi-Prüfer domain since $D$ does not possess quasi- $\star$-prime ideals, because $M^{\star}=(M V)^{v_{V}}=M^{v_{V}}=V$.

Because of the previous observations and Example 2.2 we consider with a special attention the case of $\star_{f}$-quasi-Prüfer domains.

Lemma 2.3. Let $\star$ be a semistar operation on an integral domain $D$. The following statements are equivalent:

$\left(1_{\star_{f}}\right) D$ is a $\star_{f}$-quasi-Prüfer domain.

$\left(2{\star_{f}}\right)$ Each upper to zero in $D[X]$ contains a nonzero polynomial $g \in D[X]$ with $\boldsymbol{c}(g)^{\star}=D^{\star}$.

$\left(3_{\star_{f}}\right)$ If $Q$ is an upper to zero in $D[X]$, then $\boldsymbol{c}(Q)^{\star} f=D^{\star}$.

Proof. $\left(1_{\star_{f}}\right) \Leftrightarrow\left(3_{\star_{f}}\right)$ follows from Lemma 2.1 because the property $Q \nsubseteq P[X]$, for all $P \in \mathrm{QMax}^{\star_{f}}(D)$ is equivalent to $\boldsymbol{c}_{D}(Q)^{\star_{f}}=D^{\star}$ (since each proper quasi- $\star_{f}$-ideal is contained in a quasi- $\star_{f}$-maximal).

$\left(3_{\star_{f}}\right) \Rightarrow\left(2_{\star_{f}}\right)$ is obvious.

$\left(2 \star_{\star_{f}}\right) \Rightarrow\left(1_{\star_{f}}\right)$ Let $Q$ be a prime ideal in $D[X]$ such that $Q \subseteq P[X]$, for some $P \in \mathrm{QSpec}^{\star} f(D)$. Assume $(Q \cap D)[X] \subsetneq Q$. Then we can find an upper to zero $Q_{1}$ in $D[X]$ such that $Q_{1} \subseteq Q[9$, Theorem A]. By assumption, there exists a nonzero polynomial $g \in Q_{1}$ such that $\boldsymbol{c}_{D}(g)^{\star}=D^{\star}$, hence in particular $\boldsymbol{c}_{D}\left(Q_{1}\right)^{\star}{ }_{f}=D^{\star}$ and so $\boldsymbol{c}_{D}(Q)^{\star} f=D^{\star}$. This implies that $Q \nsubseteq P[X]$, for all $P \in \mathrm{QSpec}^{\star} f(D)$ and this contradicts the assumption.

Corollary 2.4. Let $\star_{,} \star_{1}$ and $\star_{2}$ be semistar operations on an integral domain D.

(a) Assume that $\star_{1} \leq \star_{2}$. If $D$ is a $\star_{1}$-quasi-Prüfer domain then $D$ is a $a \star_{2}$ quasi-Prüfer domain.

(b) $D$ is a t-quasi-Prüfer domain if and only if $D$ is a UMt-domain.

(c) $D$ is a $\star_{f}$-quasi-Prüfer domain if and only if $D$ is a $\widetilde{\star}$-quasi-Prüfer domain. 
Proof. (a) and (b) follow easily from Lemma $2.3\left(\left(1_{\star_{f}}\right) \Leftrightarrow\left(2_{\star_{f}}\right)\right)$ and from Remark 1.2 (a). For (c) note also that $\boldsymbol{c}_{D}(g)^{\star}=D^{\star}$ if and only $\boldsymbol{c}_{D}(g) \not \subset P$ for all $P \in$ $\operatorname{QMax}^{\star} f(D)$ and that $\operatorname{QMax}^{\star} f(D)=\operatorname{QMax}^{\widetilde{\star}}(D)$ 24, Lemma 2.3 (1) and Corollary $3.5(2)]$.

Remark 2.5. For $\star=v$, we have observed in Corollary 2.4 (b) that the $t$-quasiPrüfer domains coincide with the UMt-domains, i.e., the domains such that each upper to zero in $D[X]$ is a maximal $t_{D[X]}$-ideal. There is no immediate extension to the semistar setting of the previous characterization, since in the general case we do not have the possibility to work at the same time with a semistar operation (like the $t$-operation) defined both on $D$ and on $D[X]$.

At this point it is natural to formulate the following question.

Question: Given a semistar operation of finite type $\star$ on $D$, is it possible to define in a canonical way a semistar operation of finite type $\star_{D[X]}$ on $D[X]$, such that $D$ is a $\star$-quasi-Prüfer domain if and only if each upper to zero in $D[X]$ is a quasi- $\star_{D[X]^{-}}$ maximal ideal? 1

However, we want to mention that Okabe and Matsuda [51, Definition 2.10] introduced a star-operation analog of the notion of UMt-domain: given a star operation $*$ on an integral domain $D$, they call $D$ a $*$-UMT ring if each upper to zero contains a nonzero polynomial $g \in D[X]$ with $\boldsymbol{c}_{D}(g)^{*}=D$. This notion coincides with the notion of $*_{f}$-quasi-Prüfer domain introduced above in the more general setting of the semistar operations (Lemma 2.3).

The next goal is to extend to the case of general $\star_{f}$-quasi-Prüfer domains the characterizations given in Theorem 1.1. For this purpose we need to extend some definitions to the semistar setting.

Let $D \subseteq R$ be an extension of integral domains and let $\star$ be a semistar operation on $D$. We will say that $R$ is a $\star-I N C$-extension of $D$ if whenever $Q_{1}$ and $Q_{2}$ are nonzero prime ideals of $R$ such that $Q_{1} \cap D=Q_{2} \cap D$ and $\left(Q_{1} \cap D\right)^{\star} \subsetneq D^{\star}$, then $Q_{1}$ and $Q_{2}$ are incomparable. We also say that $D$ is a $\star-I N C$-domain if each overring of $D$ is a $\star$-INC-extension of $D$. Moreover, we say that an element $u \in R$ is $\star$-primitive over $D$ if $u$ is a root of a nonzero polynomial $g \in D[X]$ with $\boldsymbol{c}_{D}(g)^{\star}=D^{\star}$.

Note that the notion of $d$-primitive (respectively, $d$-INC) extension coincides with the "classical" notion of primitive (respectively, INC) extension. It is obvious that the notions of $\star$-primitive and $\star_{f}$-primitive coincide, while $\star_{f}$-INC-extension implies $\star$-INC-extension. The converse is not true as it will be shown in the following example.

Example 2.6. Example of a $\star-I N C$ extension which is not a $\star_{f}-I N C$ extension.

Let $D, V, M$ and $\star$ be as in Example 2.2. It is easy to see that $D$ is not a $\star_{f}$-INC domain. For instance, if $R:=\pi^{-1}(k[Z])$, then $M$ is a prime ideal also in $R$ and all the maximal ideals of $R$ and the prime (non maximal) ideal $M$ of $R$ have the same trace in $D$, that is $M$. Since $M$ is a (quasi-) $\star_{f}$-maximal ideal of $D, D \hookrightarrow R$ is not a $\star_{f}$-INC extension. On the other hand $D$ is vacuously a $\star$-INC domain (the only nonzero prime of $D$ is $M$ and $M^{\star} \cap D=D$ ).

\footnotetext{
${ }^{1}$ Added in Proofs: This problem was solved by the authors in case of a stable semistar operation of finite type. The corresponding paper "Uppers to zero and semistar operations in polynomial rings" is now published in Journal of Algebra 318 (2007) 484-493.
} 
Lemma 2.7. Let $D$ be an integral domain with quotient field $K$ and let $P$ be a prime ideal of $D$. For $u \in K, D \subseteq D[u]$ satisfies INC at $P$ if and only if there exists $0 \neq g \in D[X]$ such that $\boldsymbol{c}_{D}(g) \nsubseteq P$ and $g(u)=0$.

Proof. Let $I$ be the kernel of the canonical surjective homomorphim $D[X] \rightarrow$ $D[u], X \mapsto u$. It is known that $D \subseteq D[u] \cong D[X] / I$ satisfies INC at $P$ if and only if $\boldsymbol{c}_{D}(I) \nsubseteq P$ [54, Proposition 2.0]. Suppose $\boldsymbol{c}_{D}(I) \nsubseteq P$. Choose $a \in \boldsymbol{c}_{D}(I) \backslash P$. Since $a \in \boldsymbol{c}_{D}(I)$, then there exist a finite family of polynomials $f_{1}, \ldots, f_{k} \in I$ such that $a \in \boldsymbol{c}_{D}\left(f_{1}\right)+\boldsymbol{c}_{D}\left(f_{2}\right)+\cdots+\boldsymbol{c}_{D}\left(f_{k}\right)$. Let $g:=$ $f_{1}+X^{n_{1}+1} f_{2}+X^{n_{1}+n_{2}+2} f_{3}+\cdots+X^{n_{1}+n_{2}+\cdots+n_{k-1}+k-1} f_{k}$, where $n_{i}$ is the degree of $f_{i}$. Then $g \in I, a \in \boldsymbol{c}_{D}(g)$, and $g(u)=0$. Since $a \notin P$, then $\boldsymbol{c}_{D}(g) \nsubseteq P$. Conversely, if $g(u)=0$, then $g \in I$, and hence $\boldsymbol{c}_{D}(g) \nsubseteq P$ implies $\boldsymbol{c}_{D}(I) \nsubseteq P$.

Recall from Remark 1.2 (a) that an overring $R$ of $D$ is called $t$-linked to $D$ if for each nonzero finitely generated ideal $I$ of $D,(D: I)=D$ implies $(R: I R)=R$.

Remark 2.8. The notion of $t$-linked overring can be characterized in several ways. In particular, the following statements are equivalent [12, Proposition 2.1]:

(i) $R$ is a t-linked overring to $D$.

(ii) For each nonzero finitely generated ideal I of $D, I^{t_{D}}=D$ implies $(I R)^{t_{R}}=$ $R$.

(iii) For each prime (or maximal) $t_{R}$-ideal $Q$ of $R,(Q \cap D)^{t_{D}} \subsetneq D$.

In case that $\star$ is a semistar operation on $D$, we need the following (relativized) extension of the notion of $t$-linkedness. We say that an overring $R$ of $D$ is $t$-linked to $(D, \star)$ if, for each nonzero finitely generated ideal $I$ of $D, I^{\star}=D^{\star}$ implies $(I R)^{t_{R}}=R$ [15, Section 3]. Therefore the notion of " $R$ is $t$-linked to $\left(D, t_{D}\right)$ " coincides with the "classical" notion of " $R$ is $t$-linked to $D$ ".

We collect in the following lemma some characterizations of the $t$-linkedness in the semistar setting.

Lemma 2.9. Let $\star$ be a semistar operation on an integral domain $D$ with quotient field $K$ and let $R$ be an overring of $D$. The following statements are equivalent:

(i) $R$ is a t-linked overring to $(D, \star)$.

(i $\left.i_{f}\right) R$ is a t-linked overring to $\left(D, \star_{f}\right)$.

(i) $R$ is a t-linked overring to $(D, \widetilde{\star})$.

(ii) For each nonzero ideal I of $D, I^{\star_{f}}=D^{\star}$ implies $(I R)^{t_{R}}=R$.

(iii) For each prime (or maximal) $t_{R^{-i d e a l} Q}$ of $R,(Q \cap D)^{\star_{f}} \subsetneq D^{\star}$.

(iv) For each proper $t_{R}$-ideal $J$ of $R,(J \cap D)^{\star}{ }_{f} \subsetneq D^{\star}$.

(v) $R=R^{\widetilde{\star}}(=R \cdot \mathrm{Na}(D, \star) \cap K)$.

Proof. (i) $\Leftrightarrow\left(\mathrm{i}_{f}\right) \Leftrightarrow(\widetilde{\mathrm{i}})$ because, for a nonzero finitely generated ideal $I$ of $D, I^{\star}=D^{\star}$ is equivalent to say that $I \nsubseteq P$, for all $P \in \operatorname{QMax}^{\star_{f}}(D)=\operatorname{QMax}^{\widetilde{\tau}}(D)$.

The equivalences (i) $\Leftrightarrow($ ii) $\Leftrightarrow$ (iii) $\Leftrightarrow$ (iv) are consequences of [15, Proposition 3.2].

(iii) $\Rightarrow$ (v) From the assumption it follows that, for each maximal $t$-ideal $Q$ of $R$, there exists a quasi- $\star_{f}$-maximal ideal $P$ of $D$ containing $Q \cap D$ and thus $D_{P} \subseteq$ $R_{D \backslash P} \subseteq R_{Q}$. Therefore $R \subseteq R^{\widetilde{\star}}=\bigcap\left\{R D_{P} \mid P \in \operatorname{QMax}^{\star_{f}}(D)\right\} \subseteq \bigcap\left\{R_{Q} \mid Q \in\right.$ $\left.\operatorname{Max}^{t_{R}}(R)\right\}=R$.

(v) $\Rightarrow$ (iii) Let $Q$ be a prime $t$-deal of $R$ such that $(Q \cap D)^{\star_{f}}=D^{\star}$. Therefore there exists a nonzero finitely generated ideal $I \subset Q \cap D$ such that $I^{\star_{f}}=D^{\star}$. In particular, we have $I R[X] \cap \mathcal{N}^{\star_{f}} \neq \emptyset$ and so $(I R)^{\widetilde{\star}}=I R \cdot \mathrm{Na}(D, \star) \cap K=I R[X]_{\mathcal{N}^{\star} f} \cap K=$ 
$R[X]_{\mathcal{N}^{\star} f} \cap K=R^{\widetilde{\star}}=R$. On the other hand $I R \subseteq Q$ and so $(I R)^{t_{R}} \subseteq Q^{t_{R}}=Q$. Moreover, if we denote by $\iota$ the canonical embedding of $D$ into $R$, then $*:=(\widetilde{\star})_{\iota}$ is a (semi)star operation of finite type on $R$, since $R=R^{\widetilde{\star}}=R^{*}$. Therefore $* \leq t_{R}$ and so we get a contradiction, since $R=(I R)^{\widetilde{\varkappa}}=(I R)^{*} \subseteq(I R)^{t_{R}} \subseteq Q \subsetneq R$.

Remark 2.10. Given a star operation $*$ on $D$, the property (v) of Lemma 2.9 is used in [6, page 224] for giving the definition " $R$ is $*$-linked to $D$ " (teminology used in that paper). That notion coincides with the notion of " $R$ is $t$-linked to $(D, *)$ " (terminology used here) (cf. [6, Proposition 3.2]).

Note also that, from the previous Lemma 2.9 we re-obtain in particular the equivalences stated in Remark 2.8 .

As a consequence of the previous Lemma 2.9 we deduce immediately the following two corollaries.

Corollary 2.11. Let $R$ be an overring of an integral domain $D$ with quotient field $K$. Then $R$ is a t-linked overring to $D$ if and only if $R=R^{w_{D}}\left(=R \cdot \mathrm{Na}\left(D, v_{D}\right) \cap K\right)$.

For the next statement we need to recall the notion of $\star$-valuation overring (a notion due essentially to P. Jaffard (45, page 46], see also [32, Chapters 15 and 18]). For a domain $D$ and a semistar operation $\star$ on $D$, we say that a valuation overring $V$ of $D$ is $a \star$-valuation overring of $D$ provided $F^{\star} \subseteq F V$, for each $F \in \boldsymbol{f}(D)$. Note that, by definition, the $\star$-valuation overrings coincide with the $\star_{f}$-valuation overrings. Recall that the $\star$-closure of $D$, defined by:

$$
D^{\mathrm{cl}^{\star}}:=\bigcup\left\{\left(F^{\star}: F^{\star}\right) \mid F \in \boldsymbol{f}(D)\right\}
$$

is an integrally closed overring of $D$ and, more precisely, $D^{\mathrm{cl}^{\star}}=\bigcap\{V \mid V$ is a $\star$-valuation overring of $D$ \}. Finally, recall that a valuation overring $V$ of $D$ is a $\widetilde{\star}$-valuation overring of $D$ if and only if $V$ is an overring of $D_{P}$, for some $P \in \operatorname{QMax}^{\star_{f}}(D)$. For more details on this subject and for the proofs of the results recalled above, see [51, 31, 32], 22, Proposition 3.2 and Corollary 3.6] and [24. Theorem 3.9].

Corollary 2.12. Let $\star$ be a semistar operation on an integral domain $D$ and let $V$ be a valuation overring of $D$. The following statements are equivalent:

(i) $V$ is a t-linked overring to $(D, \star)$.

(ii) $V=V^{\widetilde{\star}}$.

(iii) $V$ is a $\widetilde{\star}$-valuation overring to $D$.

Proof. Note that the $t$-operation on $V$ coincides with the $d$-operation and so (ii) $\Leftrightarrow($ iii) by [16, Lemma 2.7]. The equivalence (i) $\Leftrightarrow$ (ii) is a particular case of Lemma 2.9 (i) $\Leftrightarrow(\mathrm{v})$.

Remark 2.13. In relation with the previous corollary note that, given a semistar operation $\star$ on an integral domain $D$, it is known that each overring $R$ of $D$ is $t$-linked to $(D, \star)$ if and only if each valuation overring $V$ of $D$ is $t$-linked to $(D, \star)$ (cf. [15, Theorem 3.9] and [56, Theorem 2.15]).

Lemma 2.14. Let $\star$ be a semistar operation on an integral domain $D$. Then the following statements are equivalent.

(i) $D \subseteq K$ is a $\star_{f}$-primitive extension (or, $a \star$-primitive extension).

(ii) $D$ is a $\star_{f}$-INC-domain. 
(iii) Each t-linked overring to $(D, \star)$ is a $\star_{f}$-INC extension of $D$.

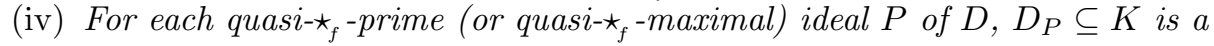
primitive extension.

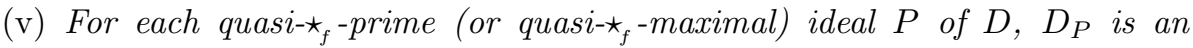
INC-domain.

(vi) For each quasi- $\star_{f}$-prime (or quasi- $-\star_{f}$-maximal) ideal $P$ of $D, D_{P}$ is a quasiPrüfer domain.

Proof. (i) $\Rightarrow$ (iv) Let $P$ be a nonzero quasi- $\star_{f}$-prime ideal of $D$. By assumption, if $0 \neq u \in K$, then there is a polynomial $0 \neq g \in D[X]$ such that $\boldsymbol{c}_{D}(g)^{\star}=D^{\star}$ and $g(u)=0$. Clearly $g \in D_{P}[X]$ and $\boldsymbol{c}_{D}(g) \nsubseteq P$. So $\boldsymbol{c}_{D_{P}}(g)=\boldsymbol{c}_{D}(g) D_{P}=D_{P}$, and thus $u$ is primitive over $D_{P}$.

(iv) $\Rightarrow$ (i) Let $0 \neq u \in K$, and let $I$ be the (nonzero) ideal of $D$ generated by the polynomials $f \in D[X]$ such that $f(u)=0$. If $\boldsymbol{c}_{D}(I)^{\star}{ }_{f}=D^{\star}$, there are nonzero polynomials $f_{1}, f_{2}, \ldots, f_{k} \in D[X]$ such that $f_{i}(u)=0$, for each $i$, and $\left(\boldsymbol{c}_{D}\left(f_{1}\right), \boldsymbol{c}_{D}\left(f_{2}\right), \ldots, \boldsymbol{c}_{D}\left(f_{k}\right)\right)^{\star}=D^{\star}$. Let $g:=f_{1}+X^{n_{1}+1} f_{2}+X^{n_{1}+n_{2}+2} f_{3}+\cdots+$ $X^{n_{1}+n_{2}+\cdots+n_{k-1}+k-1} f_{k}$, where $n_{i}$ is the degree of $f_{i}$. Then, clearly, $g(u)=0$ and $\boldsymbol{c}_{D}(g)=\left(\boldsymbol{c}_{D}\left(f_{1}\right), \boldsymbol{c}_{D}\left(f_{2}\right), \ldots, \boldsymbol{c}_{D}\left(f_{k}\right)\right)$, thus $\boldsymbol{c}_{D}(g)^{\star}=D^{\star}$. So $u$ is $\star$-primitive over $D$. In order to conclude, it remains to show that $\boldsymbol{c}(I)^{\star_{f}}=D^{\star}$. Assume that, for each $P \in \mathrm{QMax}^{\star_{f}}(D), D_{P}$ is a primitive extension, thus there is a polynomial $0 \neq h \in D_{P}[X]$ such that $h(u)=0$ and $\boldsymbol{c}_{D_{P}}(h)=D_{P}$. Let $0 \neq s \in D \backslash P$ with $s h \in D[X]$. Then $\boldsymbol{c}_{D}(s h) \nsubseteq P$ (otherwise $D_{P}=s D_{P}=s \boldsymbol{c}_{D_{P}}(h)=\boldsymbol{c}_{D_{P}}(s h)=$ $\boldsymbol{c}_{D}(s h) D_{P} \subseteq P D_{P}$, a contradiction). Clearly $s h \in I$ and so $\boldsymbol{c}_{D}(I) \nsubseteq P$ for all $P \in \operatorname{QMax}_{f}^{\star_{f}}(D)$. Therefore, $\boldsymbol{c}_{D}(I)^{\star_{f}}=D^{\star}$.

The equivalences (iv) $\Leftrightarrow(\mathrm{v}) \Leftrightarrow(\mathrm{vi})$ follow from Theorem $1.1((1) \Leftrightarrow(4) \Leftrightarrow(5))$.

(ii) $\Rightarrow$ (iii) is obvious.

(iii) $\Rightarrow$ (v) Let $P$ be a quasi- $\star_{f}$-prime of $D$, let $R$ be an overring of $D_{P}$, and let $Q_{1}$ and $Q_{2}$ are prime ideals of $R$ such that $Q_{1} \cap D_{P}=Q_{2} \cap D_{P}$, we want to show that $Q_{1}$ and $Q_{2}$ are incomparable. Let $I$ be a nonzero finitely generated ideal of $D$ with $I^{\star}=D^{\star}$. Note that $I \nsubseteq P$, since $P$ is a quasi- $\star_{f}$-ideal, and hence $D_{P}=I D_{P} \subseteq I R \subseteq R$, and so $I R=R$. Thus $(I R)^{t_{R}}=R$ and hence $R$ is $t$-linked to $(D, \star)$. By assumption, $R$ is a $\star_{f}$-INC extension of $D$ and $Q_{1} \cap D=Q_{2} \cap D \subseteq P$, with $P^{\star_{f}} \subsetneq D^{\star_{f}}=D^{\star}$, hence $Q_{1}$ and $Q_{2}$ are incomparable.

(v) $\Rightarrow$ (ii) Let $R$ be an overring of $D$, and let $Q_{1} \subsetneq Q_{2}$ be prime ideals of $R$ such that $Q_{2} \cap D$ is contained a quasi- $\star_{f}$-prime $P$ of $D$. We want to show that $Q_{1} \cap D \subsetneq Q_{2} \cap D$. If we consider the extension $D_{P} \hookrightarrow R_{D \backslash P}$ we have $Q_{1} R_{D \backslash P} \subsetneq$ $Q_{2} R_{D \backslash P} \subsetneq R_{D \backslash P}$ and $D_{P}$ is an INC-domain, by assumption. Hence $Q_{1} R_{D \backslash P} \cap$ $D_{P} \subsetneq Q_{2} R_{D \backslash P} \cap D_{P}$, and thus $Q_{1} \cap D=Q_{1} R_{D \backslash P} \cap D \subsetneq Q_{2} R_{D \backslash P} \cap D=Q_{2} \cap D$.

In Theorem 1.1 we gave several characterizations of quasi-Prüfer domains. The main goal of this section is to give a semistar analog characterization theorem for $\star_{f}$-quasi-Prüfer domains, completing the work initiated in Lemma 2.1. We start with a lemma that extends to the semistar integral closure the semistar operation versions of the Cohen-Seidenberg properties GU, INC and LO [47, page 28]. (See [6. Corollary 4.2], 7, Corollary 1.4], or [59, Theorem 3.3] for the star operation versions.)

Lemma 2.15. Let $\star$ be a semistar operation on an integral domain $D$ with quotient field $K$. Let $\bar{D}$ be the integral closure of $D($ in $K)$. Set $\widetilde{D}:=(\bar{D})^{\widetilde{\star}}$, where $\widetilde{\star}$ is the 
stable semistar of finite type of $D$ associated to $\star$, and let $\widetilde{\iota}: D \hookrightarrow \widetilde{D}$ be the canonical embedding. Set $*:=(\widetilde{\star}) \tau$.

(a) $\widetilde{D}$ coincides with the $\widetilde{\star}$-closure of $D$ (i.e., $\left.\widetilde{D}=\bigcup\left\{\left(F^{\widetilde{\star}}: F^{\widetilde{\star}}\right) \mid F \in \boldsymbol{f}(D)\right\}\right)$.

(b) The inclusion $\widetilde{\iota}: D \hookrightarrow \widetilde{D}$ verifies the properties $\widetilde{\star}-I N C, \widetilde{\star}-L O$ (i.e., for each quasi- $\widetilde{\star}$-prime ideal $P$ of $D$ there exists a*-prime of $\widetilde{D}$ such that $Q \cap D=P$ ), and $\widetilde{\star}-G U$ (i.e., if $P \subseteq P^{\prime}$ are quasi- $\widetilde{\star}$-prime ideals of $D$ and if $Q$ is a*-prime of $\widetilde{D}$ such that $Q \cap D=P$, then there exists a *-prime $Q^{\prime}$ of $\widetilde{D}$ such that $Q^{\prime} \cap D=P^{\prime}$ and $\left.Q \subseteq Q^{\prime}\right)$.

Proof. (a) It is known from [20, Example 2.1 (c.2)] and [24, Proposition 4.3] that $(\bar{D})^{\widetilde{\star}}=D^{\mathrm{cl}^{\widetilde{\star}}}=\bigcup\left\{\left(F^{\widetilde{*}}: F^{\widetilde{\star}}\right) \mid F \in \boldsymbol{f}(D)\right\}$, which is an integrally closed overring of $\bar{D}$ (and $D)$.

(b) Let $P$ be a quasi- $\widetilde{\star}$-prime ideal of $D$. Consider the prime ideal $P D[X]_{\mathcal{N}^{*}}$ and the integral extension $D[X]_{\mathcal{N}^{*}} \hookrightarrow \bar{D}[X]_{\mathcal{N}^{*}}$. By lying-over, we can find a prime ideal $\mathfrak{Q}$ in $\bar{D}[X]_{\mathcal{N}^{*}}$ such that $\mathfrak{Q} \cap D[X]_{\mathcal{N}^{\tilde{*}}}=P D[X]_{\mathcal{N}^{*}}$. Set $Q:=\mathfrak{Q} \cap \widetilde{D} \subseteq$ $\bar{D}[X]_{\mathcal{N}^{\widetilde{*}}} \cap K=(\bar{D})^{\widetilde{*}}=\widetilde{D}$. It is easy to see that $Q$ is a prime ideal of $\widetilde{D}$ such that $Q^{*}=Q^{\widetilde{\star}}=Q$ and $Q \cap D=P$. Similar arguments prove that $\widetilde{\iota}: D \hookrightarrow \widetilde{D}$ verifies $\widetilde{\star}$-INC and $\widetilde{\star}-\mathrm{GU}$.

A domain $D$ is called a Prüfer $\star-$ multiplication domain (for short, $P \star M D$ ) if each nonzero finitely generated ideal is $\star_{f}$-invertible (cf. for instance [20] and, for the case of the star operations, 40]). When $\star=v$ we have the classical notion of $\mathrm{P} v \mathrm{MD}$ (cf. for instance [30, [50] and [46]); when $\star=d$, where $d$ is the identity (semi)star operation, we have the notion of Prüfer domain [27, Theorem 22.1]. It is obvious that the notions of $\mathrm{P} \star \mathrm{MD}$ and $\mathrm{P} \star_{f} \mathrm{MD}$ coincide and it is known that they also coincide with the notion of $\mathrm{P} \approx \mathrm{MD}$ [20, Proposition 3.3]. Moreover, when $\star$ is a (semi)star operation then $D$ is a $\mathrm{P} \star \mathrm{MD}$ if and only if $D$ is a $\mathrm{P} v \mathrm{MD}$ and $\widetilde{\star}=t$ (and so $\left.\widetilde{\star}=\star_{f}=t=w\right)$ [20, Proposition 3.4]. Examples of $\mathrm{P} v$ MDs that are not $\mathrm{P} \star \mathrm{MDs}$ (for some (semi)star operation $\star$ on $D$ ) are given in [20, Example 3.4].

Theorem 2.16. Let $\star$ be a semistar operation on an integral domain $D$ with quotient field $K$. Let $\bar{D}$ be the integral closure of $D$ (in $K$ ) Then the following statements are equivalent.

$\left(1_{\star_{f}}\right) D$ is a $\star_{f}$-quasi-Prüfer domain.

$\left(4_{\star_{f}}\right) D \subseteq K$ is a $\star_{f}$-primitive extension.

$\left(5_{\star_{f}}\right) D$ is a $\star_{f}-I N C$-domain.

$\left(6_{\widetilde{\star}}\right)$ Set $\widetilde{D}=(\bar{D})^{\widetilde{\star}}$ and let $\widetilde{\iota}: D \hookrightarrow \widetilde{D}$ be the canonical embedding, then $\widetilde{D}$ is a $P(\widetilde{\star})_{\tau} M D$.

$\left(7_{\star_{f}}\right)$ Each overring $R$ of $D$ is a $\left(\star_{f}\right)_{\iota}$-quasi-Prüfer domain, where $\iota: D \hookrightarrow R$ is the canonical embedding.

$\left(8_{\star_{f}}\right)$ Every prime ideal of $\mathrm{Na}\left(D, \star_{f}\right)$ is extended from $D$.

$\left(9_{\star_{f}}\right) \mathrm{Na}\left(D, \star_{f}\right)$ is a quasi-Prüfer domain.

$\left(10_{\star_{f}}\right)$ The integral closure of $\mathrm{Na}\left(D, \star_{f}\right)$ is a Prüfer domain.

$\left(11_{\star_{f}}\right) D_{P}$ is a quasi-Prüfer domain, for each quasi- $\star_{f}$-maximal ideal (or, quasi$\star_{f}$-prime ideal) $P$ of $D$.

Moreover, if we assume that $\star$ is a (semi)star operation on $D$, then the previous conditions are also equivalent to the following: 
$\left(12_{\star_{f}}\right)$ Each t-linked overring to $\left(D, \star_{f}\right)$ is a $t$-quasi-Prüfer domain (or a UMtdomain) and each $\star_{f}$-maximal ideal of $D$ is a $t$-ideal.

$\left(13_{\star_{f}}\right) D$ is a $t$-quasi-Prüfer domain (or a UM t-domain) and each $\star_{f}$-maximal ideal of $D$ is a $t$-ideal.

$\left(14_{\star_{f}}\right) D$ is a t-quasi-Prüfer domain (or a UMt-domain) and $\widetilde{\star_{f}}=w$.

Proof. $\left(1_{\star_{f}}\right) \Rightarrow\left(4_{\star_{f}}\right)$ Let $0 \neq u \in K$, and let $\ell:=X-u$. Then $Q_{\ell}:=\ell K[X] \cap D[X]$ is a prime ideal of $D[X]$ (since $\ell \in K[X]$ is irreducible) and $Q_{\ell}$ is an upper to zero. So, by assumption, there is a $0 \neq g \in Q_{\ell}$ such that $c_{D}(g)^{\star}=D^{\star}$. Note that $g \in$ $Q_{\ell} \subseteq \ell K[X]$, so $g=\ell h$ for some $h \in K[X]$. Hence $g(u)=(\ell h)(u)=\ell(u) h(u)=0$, and thus $u$ is $\star$-primitive over $D$.

The equivalences $\left(4_{\star_{f}}\right) \Leftrightarrow\left(5_{\star_{f}}\right) \Leftrightarrow\left(11_{\star_{f}}\right)$ are proven in Lemma $2.14((\mathrm{i}) \Leftrightarrow(\mathrm{ii}) \Leftrightarrow(\mathrm{vi}))$. $\left(11_{\star_{f}}\right) \Rightarrow\left(1_{\star_{f}}\right)$ Let $\ell \in D[X]$ be a nonzero polynomial of $D[X]$, irreducible in $K[X]$, and let $Q_{\ell}:=\ell K[X] \cap D[X]$. Note that $Q_{\ell}$ is a prime ideal of $D[X], Q_{\ell}$ is an upper to zero and all upper to zero in $D[X]$ are of this form [47, Theorem 36]. It is easy to see that, for each quasi- $\star_{f}$-maximal ideal $P$ of $D$, the ideal $Q_{\ell, P}:=\ell K[X] \cap D_{P}[X]$ is a prime ideal of $D_{P}[X]$ such that $Q_{\ell, P} \cap D_{P}=(0)$ and $Q_{\ell, P} \cap D[X]=Q_{\ell}$. Since $D_{P}$ is quasi-Prüfer, $Q_{\ell, P}$ contains a polynomial $h \in D_{P}[X]$ such that $\boldsymbol{c}_{D_{P}}(h) \nsubseteq P D_{P}$ (Theorem $1.1(1) \Rightarrow(2)$ ). Choose $s \in D \backslash P$ with $s h \in D[X]$. Note that $s h \in Q_{\ell, P} \cap D[X]=Q_{\ell}$ and that $s \boldsymbol{c}_{D}(h)=\boldsymbol{c}_{D}(s h) \nsubseteq \mathbb{P}$, because $\boldsymbol{c}_{D_{P}}(s h) \nsubseteq P D_{P}$. Since the last property holds for each quasi- $\star_{f}$-maximal ideal $P$ of $D$, then $\boldsymbol{c}_{D}\left(Q_{\ell}\right)^{\star_{f}}=D^{\star}$. We conclude that $D$ is a $\star_{f}$-quasi-Prüfer domain by Lemma 2.3 .

$\left(1_{\star_{f}}\right) \Rightarrow\left(8_{\star_{f}}\right)$ Suppose that $D$ is a $\star_{f}$-quasi-Prüfer domain, and let $\mathfrak{Q}$ be a prime ideal of $\mathrm{Na}(D, \star)$. Then there is a prime ideal $Q$ of $D[X]$ such that $\mathfrak{Q}=$ $Q N a(D, \star)=Q D[X]_{\mathcal{N} \star}$ and so $Q \cap \mathcal{N}^{\star}=\emptyset$. Let $P:=Q \cap D$. If $P[X] \subsetneq Q$, pick $q \in Q \backslash P[X]$, and let $Q_{1}$ be an upper to zero in $D[X]$ such that $q \in Q_{1} \subseteq Q[9$, Theorem A]. Since $D$ is a $\star_{f}$-quasi-Prüfer domain and $Q_{1}$ is an upper to zero, there is a nonzero polynomial $g \in Q_{1}$ such that $\boldsymbol{c}_{D}(g)^{\star}=D^{\star}$, and hence $g \in \mathcal{N}^{\star} \cap Q$, a contradiction. So $Q=P[X]$, and thus $\mathfrak{Q}=P \mathrm{Na}(D, \star)$.

$\left(8_{\star_{f}}\right) \Rightarrow\left(1_{\star_{f}}\right)$ Suppose that $D$ is not a $\star_{f}$-quasi-Prüfer domain. By Lemma 2.3 $\left(\left(1_{\star_{f}}\right) \Leftrightarrow\left(2_{\star_{f}}\right)\right)$ then there is an upper to zero $Q$ in $D[X]$ such that $Q \cap \mathcal{N}^{\star}=\emptyset$. Hence $Q D[X]_{\mathcal{N}^{\star}}=Q \mathrm{Na}(D, \star)$ is a proper prime ideal of $\mathrm{Na}(D, \star)$. Note that $Q \mathrm{Na}(D, \star) \neq P \mathrm{Na}(D, \star)$ for all nonzero prime ideals $P$ of $D$, since $Q$ is an upper to zero. This fact contradicts the assumption $\left(8_{\star_{f}}\right)$.

$\left(9_{\star_{f}}\right) \Leftrightarrow\left(10_{\star_{f}}\right)$ follows from Theorem $1.1((1) \Leftrightarrow(6))$.

$\left(9_{\star_{f}}\right) \Rightarrow\left(11_{\star_{f}}\right)$ Let $P$ be a quasi- $\star_{f}$-maximal ideal of $D$. Then $P \mathrm{Na}(D, \star)$ is a maximal ideal of $\mathrm{Na}(D, \star)[24$, Proposition $1.3(3)]$. Hence $\mathrm{Na}\left(D_{P}\right)=D_{P}[X]_{P D_{P}[X]}$ $=\left(D[X]_{\mathcal{N}^{\star}}\right)_{P D[X]_{\mathcal{N} *}}=\mathrm{Na}(D, \star)_{P \mathrm{Na}(D, \star)}$ (cf. also [24, Theorem 3.8]). Since we are assuming that $\mathrm{Na}(D, \star)$ is quasi-Prüfer, then $\mathrm{Na}(D, \star)_{P \mathrm{Na}(D, \star)}=\mathrm{Na}\left(D_{P}\right)$ is quasiPrüfer and thus $D_{P}$ is a quasi-Prüfer domain (by Theorem $1.1((9) \Rightarrow(1)$ ).

$\left(11_{\star_{f}}\right) \Rightarrow\left(9_{\star_{f}}\right)$ Let $Q \in \operatorname{Max}(\mathrm{Na}(D, \star))$. We know that $Q=P \mathrm{Na}(D, \star)$, for some $P \in \operatorname{QMax}^{\star} f(D)$ and that $\mathrm{Na}(D, \star)_{Q}=\mathrm{Na}\left(D_{P}\right)$ [24, Proposition 1.3]. Therefore, if $D_{P}$ is quasi-Prüfer, then $\mathrm{Na}(D, \star)_{Q}=\mathrm{Na}\left(D_{P}\right)$ is quasi-Prüfer (Theorem 1.1 $(1) \Rightarrow(9)$ ). Thus $\mathrm{Na}(D, \star)$ is quasi-Prüfer (Theorem $1.1(11) \Rightarrow(1)$ ).

$\left(1_{\star_{f}}\right) \Rightarrow\left(7_{\star_{f}}\right)$ Let $R$ be an overring to $\left(D, \star_{f}\right)$ and, for simplicity of notation, set $*:=\left(\star_{f}\right)_{\iota}$. The property $\left(7_{\star_{f}}\right)$ holds if we show that $R_{Q}$ is a quasi-Prüfer domain 
for all $Q \in \operatorname{QMax}^{*}(R)$, since we already proved that $\left(1_{\star_{f}}\right) \Leftrightarrow\left(11_{\star_{f}}\right)$. Note that the prime ideal $P:=Q \cap D$ is such that $P \subseteq P^{\star_{f}} \cap D \subseteq Q^{\star_{f}} \cap D=\left(Q^{\star_{f}} \cap\right.$ $R) \cap D=\left(Q^{*} \cap R\right) \cap D=Q \cap D=P$. Since $P$ is a quasi- $\star_{f}$-prime ideal of $D$ and, by assumption, $D$ is a $\star_{f}$-quasi-Prüfer domain, then $D_{P}$ is quasi-Prüfer (by $\left(1_{\star_{f}}\right) \Rightarrow\left(11_{\star_{f}}\right)$ ). Therefore $R_{Q}$, which is an overring of $D_{P}$, is also quasi-Prüfer (Theorem $1.1(1) \Rightarrow(7)$ ).

$\left(7_{\star_{f}}\right) \Rightarrow\left(1_{\star_{f}}\right)$ is trivial.

$\left(1_{\star_{f}}\right) \Rightarrow\left(6_{\widetilde{\star}}\right)$ We already proved that $\left(1_{\star_{f}}\right)$ is equivalent to $\left(9_{\star_{f}}\right)$. Therefore we can assume that $\mathrm{Na}\left(D, \star_{f}\right) \quad(=\mathrm{Na}(D, \widetilde{\star})$ by [24, Corollary 3.7]) is a quasiPrüfer domain, i.e., the integral closure $\overline{\mathrm{Na}(D, \widetilde{\star})}$ of $\mathrm{Na}(D, \widetilde{\star})$ in $K(X)$ is a Prüfer domain. Note that $\overline{\operatorname{Na}(D, \widetilde{\star})}=\bar{D}[X]_{\mathcal{N}^{\tilde{*}}}$, where $\mathcal{N}^{\widetilde{\star}}=\mathcal{N}^{\star_{f}}=\{g \in D[X] \mid g \neq$ 0 and $\left.\boldsymbol{c}_{D}(g)^{\widetilde{\star}}=D^{\widetilde{\star}}\right\}$. For the sake of simplicity, set $*:=(\widetilde{\star})_{\widetilde{\tau}}$. Clearly $*$ is a stable (semi)star operation of finite type on $\widetilde{D}$. Moreover $\operatorname{Na}(\widetilde{D}, *)=\widetilde{D}[X]_{\tilde{\mathcal{N}}}$, where $\widetilde{\mathcal{N}}:=\mathcal{N}^{*}=\left\{h \in \widetilde{D}[X] \mid h \neq 0\right.$ and $\left.\boldsymbol{c}_{\widetilde{D}}(h)^{*}=\widetilde{D}\right\}$. Since it is clear that $\mathcal{N}^{\widetilde{*}}$ is also a multiplicative set in $\widetilde{D}[X]$ and that $\mathcal{N}^{\widetilde{*}} \subseteq \widetilde{\mathcal{N}}$, then $\overline{\mathrm{Na}(D, \widetilde{\star})} \subseteq \mathrm{Na}(\widetilde{D}, *)$ and so $\mathrm{Na}(\widetilde{D}, *)$ is a Prüfer domain [27, Theorem 26.1]. By [20, Theorem 3.1 (i) $\Leftrightarrow($ iii)], this is equivalent to say that $\widetilde{D}$ is a $\mathrm{P} * \mathrm{MD}$.

$\left(6_{\widetilde{夭}}\right) \Rightarrow\left(10_{\star_{f}}\right)$ With the notation used in the proof of $\left(1_{\star_{f}}\right) \Rightarrow\left(6_{\widetilde{夭}}\right)$, the present hypothesis is equivalent to assume that $\mathrm{Na}(\widetilde{D}, *)$ is a Prüfer domain. The conclusion will trivially follow if we show that $\overline{\operatorname{Na}(D, \widetilde{\star})}=\mathrm{Na}(\widetilde{D}, *)$, i.e., $\bar{D}[X]_{\mathcal{N}^{\star}}=\widetilde{D}[X]_{\widetilde{\mathcal{N}}}$.

Note that $\mathcal{N}^{\widetilde{*}}=D[X] \backslash \bigcup\left\{P[X] \mid P \in \operatorname{QMax}^{\widetilde{*}}(D)\right\}, \widetilde{\mathcal{N}}=\widetilde{D}[X] \backslash \bigcup\{Q[X] \mid Q \in$ $\left.\operatorname{Max}^{*}(\widetilde{D})\right\}$, and $\bar{D}[X]_{\mathcal{N}^{*}} \subseteq \widetilde{D}[X]_{\widetilde{\mathcal{N}}}$. By Lemma 2.15 (b) the natural embedding $\widetilde{\iota}: D \hookrightarrow \widetilde{D}$ verifies $\widetilde{\star}$-LO, $\widetilde{\star}$-INC and $\widetilde{\star}$-GU. It is not difficult to see that a prime ideal $Q$ of $\widetilde{D}$ belongs to $\operatorname{Max}^{*}(\widetilde{D})$ if and only if $Q \cap D$ belongs to $\operatorname{QMax}^{\widetilde{*}}(D)$.

As a matter of fact, let $Q$ be a prime ideal in $\widetilde{D}$. Assume that $P:=Q \cap D \in$ $\operatorname{QMax}^{\widetilde{\star}}(D)$. By $\widetilde{\star}-\mathrm{LO}$ we can assume that $Q$ is a $*$-prime in $\widetilde{D}$. Let $M \in \operatorname{Max}^{*}(\widetilde{D})$, such that $M \supsetneq Q$. By $\widetilde{\star}$-INC we have $M \cap D \supsetneq Q \cap D=P$. Therefore $M \cap D \subseteq$ $(M \cap D)^{\widetilde{\star}} \cap \tilde{D}=\left(M^{\widetilde{\star}} \cap D^{\widetilde{\star}}\right) \cap D \subseteq\left(M^{\widetilde{\star}} \cap \widetilde{D}\right) \cap D=\left(M^{*} \cap \widetilde{D}\right) \cap D=M \cap \bar{D}$ and so we reach a contradiction (i.e., $P$ is not in $\left.\operatorname{QMax}^{\widetilde{*}}(D)\right)$. Conversely, let $Q \in \operatorname{Max}^{*}(\widetilde{D})$ and assume that $P:=Q \cap D \subsetneq P^{\prime}$, for some prime ideal $P^{\prime}$ of $D$ such that $P^{\prime}=P^{\prime \widetilde{\star}} \cap D \in \operatorname{QMax}^{\widetilde{\star}}(D)$. By $\widetilde{\star}$-GU, there exists a $*$-prime ideal $Q^{\prime}$ of $\widetilde{D}$ such that $Q^{\prime} \cap D=Q$ and $Q \subsetneq Q^{\prime}$ and this is a contradiction.

From the fact that a prime ideal $Q$ of $\widetilde{D}$ belongs to $\operatorname{Max}^{*}(\widetilde{D})$ if and only if $Q \cap D$ belongs to $\operatorname{QMax}^{\widetilde{ }}(D)$, we deduce that the ideals of $\bar{D}[X]$ that are maximal with the property of being disjoints from $\mathcal{N}^{\widetilde{*}}$ are the ideals $\left\{(Q \cap \bar{D})[X] \mid Q \in \operatorname{Max}^{*}(\widetilde{D})\right\}$. From this fact it follows easily that $\bar{D}[X]_{\mathcal{N}^{\tilde{*}}}=\widetilde{D}[X]_{\widetilde{\mathcal{N}}}$

$\left(13_{\star_{f}}\right) \Leftrightarrow\left(14_{\star_{f}}\right)$ The second part of condition $\left(13_{\star_{f}}\right)$ implies that $\operatorname{Max}^{\star_{f}}(D)=$ $\operatorname{Max}^{t}(D)$ and so $\widetilde{\star}=w$. Conversely, if $\widetilde{\star}=w$, then $\operatorname{Max}^{\star_{f}}(D)=\operatorname{Max}^{\widetilde{\star}}(D)=$ $\operatorname{Max}^{w}(D)=\operatorname{Max}^{t}(D)$, and so each quasi- $\star_{f}$-ideal maximal of $D$ is a $t$-ideal.

$\left(1_{\star_{f}}\right) \Rightarrow\left(13_{\star_{f}}\right)$ Under the present assumptions $\star_{f}$ is a (semi)star operation of finite type on $D$, then $\star_{f} \leq t$ (essentially by [27, Theorem 34.1 (4)]). Therefore if $D$ is a $\star_{f}$-quasi-Prüfer domain then $D$ is also a $t$-quasi-Prüfer domain (Corollary 2.4 (a)). Let $P$ be a $\star_{f}$-maximal ideal of $D$. Since we already proved that $\left(1_{\star_{f}}\right) \Rightarrow$ 
$\left(11_{\star_{f}}\right)$, then $D_{P}$ is a quasi-Prüfer domain. By Corollary $1.3((1) \Rightarrow(13)) P D_{P}$ is a $t$-ideal in $D_{P}$ and thus $P=P D_{P} \cap D$ is a $t$-ideal of $D$ [46, Lemma 3.17].

$\left(13_{\star_{f}}\right) \Rightarrow\left(10_{\star_{f}}\right)$ Since each $\star_{f}$-maximal ideal of $D$ is a $t$-ideal, then necessarily $\operatorname{Max}^{\star_{f}}(D)=\operatorname{Max}^{t}(D)$ and hence $\mathcal{N}^{\star}=\mathcal{N}^{\star_{f}}=\mathcal{N}^{t}=\mathcal{N}^{v}$. Thus $\operatorname{Na}(D, \star)=$ $\mathrm{Na}(D, v)$ and $\mathrm{Na}(D, v)$ has Prüfer integral closure by [21, Theorem 2.5], since $D$ is an UMt-domain (=t-quasi-Prüfer domain).

$\left(1_{\star_{f}}\right) \Rightarrow\left(12_{\star_{f}}\right)$ Let $R$ be a $t$-linked overring to $\left(D, \star_{f}\right)$, then $R=R^{\widetilde{\star}}$ (Lemma 2.9 $((\mathrm{i}) \Rightarrow(\mathrm{v})))$. Let $\iota: D \hookrightarrow R$ be the canonical embedding, then $(\widetilde{\star}) \iota$ is a (semi)star operation of finite type on $R$. Since $D$ is a $\star_{f}$-quasi-Prüfer domain or, equivalently, a $\widetilde{\star}$-quasi-Prüfer domain (Corollary $2.4(\mathrm{c})$ ) then, by $\left(1_{\star_{f}}\right) \Leftrightarrow\left(7_{\star_{f}}\right), R$ is a $(\widetilde{\star})_{\iota^{-}}$ quasi-Prüfer domain. Since in the present situation $(\widetilde{\star})_{\iota} \leq t_{R}$ then $R$ is also a

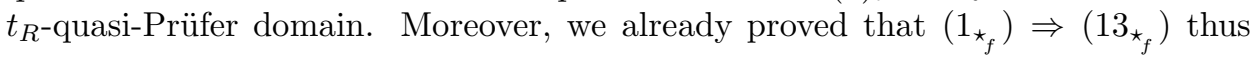
each $\star_{f}$-maximal ideal of $D$ is a $t$-ideal.

$\left(12_{\star_{f}}\right) \Rightarrow\left(13_{\star_{f}}\right)$ is trivial.

Let $\star$ be a semistar operation on an integral domain $D$. Recall that a $\mathrm{P} \star \mathrm{MD}$ $D$ can be characterized by the fact that $D_{P}$ is a valuation domain for each $P \in$ $\operatorname{QMax}^{\star_{f}}(D)$ [20, Theorem 3.1]. Thus, since a valuation domain is trivially quasiPrüfer, a $\mathrm{P} \star \mathrm{MD}$ is a $\star_{f}$-quasi-Prüfer domain by Theorem $2.16\left(\left(1_{\star_{f}}\right) \Leftrightarrow\left(11_{\star_{f}}\right)\right)$. This fact generalizes the well known property that a $\mathrm{P} v \mathrm{MD}$ is a UMt domain (Corollary 2.4 (b)). However, a $\mathrm{P} \star \mathrm{MD}$ need not be integrally closed (cf. 20, Example 3.10]), while being a $\mathrm{P} v \mathrm{MD}$ is equivalent to being an integrally closed UMt domain [42, Proposition 3.2]. The next corollary gives an appropriate generalization of the previous result to the case of semistar operations.

Corollary 2.17. 20, Theorem 3.2] Let $\star$ be a semistar operation on an integral domain $D$ with quotient field $K$. Then the following statements are equivalent.

(i) $D$ is a $P \star M D$.

(ii) $D$ is a $\star_{f}$-quasi-Prüfer domain and $D_{P}$ is integrally closed for all $P \in$ $\operatorname{QMax}^{\star} f(D)$.

(iii) $D$ is a $\star_{f}$-quasi-Prüfer domain and $D^{\widetilde{\star}}$ is integrally closed.

Proof. The implication (i) $\Rightarrow$ (ii) was already proved just before the statement of Corollary 2.17.

(ii) $\Rightarrow$ (iii) This follows from [47, Theorem 52] because $D^{\widetilde{\star}}=\bigcap\left\{D_{P} \mid P \in\right.$ $\left.\mathrm{QMax}^{\star_{f}}(D)\right\}$ and each $D_{P}$ is integrally closed, by assumption.

(iii) $\Rightarrow$ (i) Let $\iota: D \hookrightarrow D^{\widetilde{\star}}$ be the canonical embedding and set $*:=(\widetilde{\star})$ (thus $E^{*}=E^{\widetilde{\star}}$ for all $E \in \overline{\boldsymbol{F}}\left(D^{\widetilde{\star}}\right)(\subseteq \overline{\boldsymbol{F}}(D))$. Then $\mathrm{Na}\left(D, \star_{f}\right)=\mathrm{Na}(D, \widetilde{\star})=\mathrm{Na}\left(D^{\widetilde{\star}}, *\right)$ 24, Corollary 3.5]. On the other hand $\mathrm{Na}\left(D^{\widetilde{\star}}, *\right)$ is integrally closed, because $D^{\widetilde{\star}}$ is integrally closed by assumption, and $\mathrm{Na}\left(D, \star_{f}\right)$ is quasi-Prüfer domain by Theorem $2.17\left(\left(1_{\star_{f}}\right) \Leftrightarrow\left(9_{\star_{f}}\right)\right)$. Putting these two pieces of information together we deduce that $\mathrm{Na}\left(D, \star_{f}\right)$ is a Prüfer domain and thus $D$ is a $\mathrm{P} \star_{f} \mathrm{MD}$ (or, a $\mathrm{P} \star \mathrm{MD}$ ) by [20, Theorem 3.1].

The following corollary follows immediately from Theorem $2.16\left(\left(1_{\star_{f}}\right) \Leftrightarrow\left(6_{\widetilde{\star}}\right)\right)$ and from [20, Proposition 3.4].

Corollary 2.18. (cf. [59, Theorem 4.2]) Let $D$ be an integral domain with quotient field $K$. Set $\widetilde{D}=(\bar{D})^{w_{D}}$ and let $\widetilde{\iota}: D \hookrightarrow \widetilde{D}$ be the canonical embedding. The following statements are equivalent: 
(i) $D$ is a $U M t_{D}$-domain.

(ii) $\widetilde{D}$ is a $P\left(w_{D}\right) \tau M D$.

(iii) $\widetilde{D}$ is a $P v_{\widetilde{D}} M D$ and $\left(w_{D}\right)_{\tau}=w_{\widetilde{D}}=t_{\widetilde{D}}$.

We have already mentioned in Remark 1.2 (d) the interesting open problem of establishing whether the integral closure of a UMt-domain is a P $v$ MD. For a negative answer to this problem we need examples of integral domains $D$ such that the integral closure $\bar{D}$ is not $t$-linked to $D$ (Remark 1.2 (a)). This is not an easy task, even in a general situation. Note that the integral closure $\bar{D}$ is $t$-linked to $D$ if $D$ is one-dimensional [12, Corollary 2.7] or if $D$ is quasi-coherent (e.g., $D$ is Noetherian) [12, Corollary 2.14 (a)]. A first class of examples of integral domains of dimension $\geq 3$ such that the integral closure $\bar{D}$ is not $t$-linked to $D$ was given in [13. Example 4.1]. The 2-dimensional case was left open in that paper. A first example in dimension two was given by Dumitrescu [14], using the $A+X B[X]$ constructions. We give next another example of this type.

Example 2.19. A quasi-local strong Mori non Noetherian 2-dimensional UMtdomain $D$ such that $\bar{D}$ is not t-linked to $D$ but still $\bar{D}$ is a $P v_{\bar{D}} M D$.

For this purpose we use a construction due to Heinzer, Ohm and Pendleton 35. Example 2.10]. Let $K$ be a field, $X, Y$ indeterminates over $K$, let $V$ be the $X$-adic valuation ring of $K(X, Y)$, i.e. $V:=K(Y)[X]_{(X)}$, and let $M_{X}:=X K(Y)[X]_{(X)}$ be the maximal ideal of $V$ (hence $\left.V=K(Y)+M_{X}\right)$. Also, let $D_{1}:=K[X, Y]_{(X, Y)}$, $M_{1}:=(X, Y) K[X, Y]_{(X, Y)}, k_{T}:=K\left(Y+\frac{1}{Y}\right) \subsetneq K(Y)$ and set $T:=k_{T}+M_{X}$, and $D:=T \cap D_{1}$. Note that if we consider the Krull overring $R:=D_{1}[1 / X]=$ $\bigcap\left\{D_{1 P_{1}} \mid P_{1} \neq\left(M_{X} \cap D_{1}\right)\right.$ with $P_{1}$ height 1 prime ideal of $\left.D_{1}\right\}$ of $D$ (and of $D_{1}$ ) [26. Corollary 1.5 and Proposition 3.15], then we also have $D=R \cap T$ (and $\left(D_{1}=R \cap V\right)$.

(a) $T$ is a 1-dimensional Noetherian pseudo-valuation domain (or, PVD) with maximal ideal $M_{X}$ and associate valuation overring $V$. Moreover the integral closure $\bar{T}$ of $T$ coincides with $V$.

Note that $k_{T} \hookrightarrow K(Y)$ is a finite extension, since $Y$ is a root of the polynomial $Z^{2}-\left(\left(Y^{2}+1\right) / Y\right) Z+1$ in the indeterminate $Z$ with coefficients in $k_{T}$. The conclusion follows from [33, Theorem 3.1 and Corollary 3.4].

(b) Let $Q:=M_{X} \cap D=X K[X, Y]_{(X, Y)}=M_{X} \cap D_{1}$. Then $D_{1} \subsetneq V=\left(D_{1}\right)_{Q}$ and that $D$ and $D_{1}$ have a common prime ideal, i.e., $Q$. In particular, the map $H_{1} \mapsto H:=H_{1} \cap D$ establishes a $1-1$ correspondence betweeen the prime ideals of $D_{1}$ not containing $Q$ and the prime ideal of $D$ not containing $Q$ and, moreover, $D_{H}=\left(D_{1}\right)_{H_{1}}$. For the remaining localization of $D$ at the prime $Q$, we have $D_{Q}=T \subsetneq\left(D_{1}\right)_{Q}$.

After remarking that $Q$ is a common ideal of $D$ and $D_{1}$, the first part follows from the general properties of the pullback diagrams [17, Claim (c) in the proof of Theorem 1.4]. The last statement is proved in [35. Lemma, page 152].

(c) $D$ is a quasi-local domain with maximal ideal $M:=M_{1} \cap D$, with complete integral closure equal to $D_{1}$ and $\operatorname{dim}(D)=2$.

The first part of the statement is proved in [35. Example 2.10, page 152]. The reamining part follows from the fact that $D$ and $D_{1}$ have $Q$ as common ideal [27, Lemma 26.5] and from the fact that $\operatorname{dim}\left(D_{1}\right)=2$.

(d) $D$ is a strong Mori domain with $\operatorname{dim}^{t}(D)=1$, 
Let $\Lambda_{1}:=\left\{P_{1} \in D_{1} \mid P_{1}\right.$ is a height 1 prime ideal of $\left.D_{1}, P_{1} \neq Q\right\}$ (resp., $\Lambda:=$ $\{P \in D \mid P$ is a height 1 prime ideal of $D, P \neq Q\}$ ). From (b) and from the presentations $D=\left(\bigcap\left\{D_{P} \mid P \in \Lambda\right\}\right) \cap D_{Q}=\left(\bigcap\left\{D_{1 P_{1}} \mid P_{1} \in \Lambda_{1}\right\}\right) \cap D_{Q}=\left(\bigcap\left\{D_{1 P_{1}} \mid\right.\right.$ $\left.\left.P_{1} \in \Lambda_{1}\right\}\right) \cap T \subsetneq\left(\bigcap\left\{D_{1 P_{1}} \mid P_{1} \in \Lambda_{1}\right\}\right) \cap V=\left(\bigcap\left\{D_{1 P_{1}} \mid P_{1} \in \Lambda_{1}\right\}\right) \cap D_{1 Q}=D_{1}$ we deduce that $D$ is a Mori domain (in particular, $t=v$ ) [4, Construction 4.1 and Theorem 4.3]. Obviously, all the height 1 prime ideals of $D$ are $t$-ideals of $D$, but the maximal ideal $M$ is not a $t$-ideal (or a $v$-ideal) of $D$ [4, Theorem 4.3 (f)]. Henceforth $\operatorname{dim}^{t}(D)=1$ and $\operatorname{Max}^{t}(D)=\{P \in \operatorname{Spec}(D) \mid \operatorname{ht}(P)=1\}$. Furthermore, note that $D_{P}$ is Noetherian for all $P \in \operatorname{Max}^{t}(D)$ and each nonzero element of $D$ lies in only finitely many maximal $t$-ideals of $D$ (because this property holds in $D_{1}$ ) 4, Theorem 4.3 (a)]. Therefore, by [61, Theorem 1.9], $D$ is a strong-Mori domain (i.e., $D$ verifies the acc on the $w$-ideals [60]) and, clearly, $D=\bigcap\left\{D_{P} \mid P \in \operatorname{Max}^{t}(D)\right\}=D^{w}$.

(e) $D$ is a UMt-domain.

By $\left(\right.$ d) $\operatorname{dim}^{t}(D)=1$, then $D$ is a UMt-domain by [7, Corollary $\left.3.2((6) \Rightarrow(1))\right]$.

(f) The integral closure $\bar{D}$ of $D$ coincides with $\left(W_{1} \cap W_{2}\right)+Q$, where $W_{1}:=$ $K[Y]_{(Y)}$ and $W_{2}:=K\left[\frac{1}{Y}\right]_{\left(\frac{1}{Y}\right)}$. Therefore $\bar{D} \subsetneq D_{1}, \bar{D} / Q=W_{1} \cap W_{2}$ is a semi-quasi-local PID with two maximal ideals and $D_{1} / Q=W_{1}$.

The first part of the statement is proved in [35, Example 2.10, page 152]. The remaining part is an easy consequence of the first part [47, Theorem 107].

The following three statements are immediate consequences of (f).

(g) $\bar{D}$ is semi-quasi-local with two maximal $\bar{M}_{1}$ and $\bar{M}_{2}$ such that $\bar{M}_{1} \cap D=$ $\bar{M}_{2} \cap D=M$ and $\operatorname{ht}\left(\bar{M}_{1}\right)=\operatorname{ht}\left(\bar{M}_{2}\right)=2$. Moreover, $\bar{D}_{\bar{M}_{1}} / Q \bar{D}_{\bar{M}_{1}}=W_{1}$ and $\bar{D}_{\bar{M}_{2}} / Q \bar{D}_{\bar{M}_{2}}=W_{2}$.

(h) The only prime ideals of $\bar{D}$ containing $X$ (i.e., the prime ideal $Q=X D_{1}$ ) are $\bar{M}_{1}, \bar{M}_{2}$ and, obviously, $Q$.

(i) $D$ and $\bar{D}$ have a common prime ideal $Q$, then -as in point (b)- the map $\bar{H} \mapsto H:=\bar{H} \cap D$ establishes a $1-1$ correspondence betweeen the prime ideals of $\bar{D}$ not containing $Q$ and the prime ideal of $D$ not containing $Q$ and, moreover, $D_{H}=\bar{D}_{\bar{H}}$. Furthermore, as a consequence of (a) and (b), $\bar{D}_{Q}=V$.

(j) $\bar{D} \subsetneq(\bar{D})^{w_{D}}=D_{1}$. Therefore $\bar{D}$ is not $t$-linked to $D($ Lemma $2.9((\mathrm{i}) \Rightarrow(\mathrm{v})))$ and $D$ is not Noetherian.

As already remarked in [35, Example 2.10, page 152], we have $(\bar{D})^{w_{D}}=\bigcap\{\bar{D}$. $\left.D_{P} \mid P \in \operatorname{Max}^{t}(D)\right\}=\bar{D}_{D \backslash Q} \cap\left(\bigcap\left\{\bar{D}_{D \backslash P} \mid P \in \Lambda\right\}\right)=V \cap\left(\bigcap\left\{D_{1 P_{1}} \mid P_{1} \in\right.\right.$ $\left.\left.\Lambda_{1}\right\}\right)=V \cap R=D_{1}$ (cf. also [7, Theorems 1.3 and 3.1]). The claim that $D$ is not Noetherian is a consequence of the fact that $\bar{D} \neq D_{1}$ and that, by (c), $D_{1}$ is the complete integral closure of $D$.

Set $A:=W_{1} \cap W_{2}, B:=W_{1}$, and let $\mathfrak{m}_{1}, \mathfrak{m}_{2}$ be the maximal ideals of $A$, with $A_{\mathfrak{m}_{1}}=W_{1}$ and $A_{\mathfrak{m}_{2}}=W_{2}$ (cf. (f)). By the previous considerations, we have the following pullback diagrams of canonical homomorphisms: 


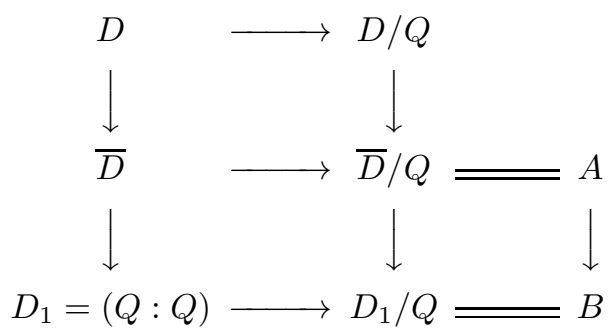

(k) $\bar{D}$ is a $\mathrm{P} v_{\bar{D}} \mathrm{MD}$.

We claim that for each prime $t$-ideal $\mathfrak{p}$ of $A$ either $A_{\mathfrak{p}}$ is a valuation domain and $B_{A \backslash \mathfrak{p}}$ is a field or there exists a finitely generated ideal $\mathfrak{f}$ of $A, \mathfrak{f} \subseteq \mathfrak{p}$ such that $(A: \mathfrak{f}) \cap A_{\mathfrak{p}}=A$. As a matter of fact, by (f), $A$ is a PID with $\operatorname{Max}(A)=\left\{\mathfrak{m}_{1}, \mathfrak{m}_{2}\right\}$, then the set of prime $t$-ideals of $A$ coincides with $\operatorname{Max}(A)$. Clearly, $A_{\mathfrak{m}_{2}}=W_{2}$ and $B_{A \backslash \mathfrak{m}_{2}}=\left(W_{1}\right)_{A \backslash \mathfrak{m}_{2}}$ is the quotient field of $B$ (and of $A$ ). On the other hand $A_{\mathfrak{m}_{1}}=W_{1}=B_{A \backslash \mathfrak{m}_{1}}$, but if $\mathfrak{m}_{1}=\pi A$, then $(A: \pi A) \cap A_{\pi A}=\pi^{-1} A \cap A_{\pi A}=A$, since $A \cap \pi A_{\pi A}=\pi A$. Now the statement follows from [41, Theorems 4.8 and 5.2].

Remark 2.20. (a) With the notation of Theorem 2.16 let $D \stackrel{\bar{\iota}}{\hookrightarrow} \bar{D}, \bar{D} \stackrel{j}{\hookrightarrow} \widetilde{D}$ and $D \stackrel{\widetilde{\iota}}{\hookrightarrow} \widetilde{D}$ be the canonical embeddings and so $\widetilde{\iota}=j \circ \bar{\iota}$. Note that the statement $\left(6_{\widetilde{\star}}\right)$ is equivalent to each of the following:

$\left(6_{\widetilde{\star}}^{\prime}\right) \widetilde{D}$ is a $P v_{\widetilde{D}} M D$ and $(\widetilde{\star})_{\widetilde{\iota}}=w_{\widetilde{D}}=t_{\widetilde{D}}$.

$\left(\overline{6}_{\widetilde{\star}}\right) \bar{D}$ is a $P(\widetilde{\star})_{\bar{\tau}} M D$.

$\left(\overline{6}_{\widetilde{\star}}^{\prime}\right) \bar{D}$ is a $P\left(v_{\widetilde{D}}\right)^{j} M D$ and $(\widetilde{\star})_{\widetilde{\iota}}=w_{\widetilde{D}}=t_{\widetilde{D}}$.

The equivalence $\left(6_{\widetilde{\star}}\right) \Leftrightarrow\left(6_{\widetilde{\star}}^{\prime}\right)$ follows immediately from [20, Proposition 3.4], since $(\widetilde{\star})_{\widetilde{\iota}}$ is a (semi)star operation on $\widetilde{D}$.

$\left(6_{\widetilde{\star}}\right) \Rightarrow\left(\overline{6}_{\widetilde{\star}}\right)$ Set

$$
\begin{aligned}
& \mathcal{N}^{\widetilde{\star}}=\left\{g \in D[X] \mid g \neq 0 \text { and } \boldsymbol{c}_{D}(g)^{\widetilde{\star}}=D^{\widetilde{\star}}=\widetilde{D}\right\} \text {, } \\
& \overline{\mathcal{N}}:=\mathcal{N}^{(\widetilde{\star})_{\bar{\tau}}}=\left\{\ell \in \bar{D}[X] \mid \ell \neq 0 \text { and } \boldsymbol{c}_{\bar{D}}(\ell)^{(\widetilde{\star})_{\bar{\tau}}}=\bar{D}^{(\widetilde{\star})_{\bar{\tau}}}=\widetilde{D}\right\}, \\
& \widetilde{\mathcal{N}}:=\mathcal{N}^{(\widetilde{\star})_{\tilde{\iota}}}=\left\{h \in \widetilde{D}[X] \mid h \neq 0 \text { and } \boldsymbol{c}_{\widetilde{D}}(h)^{(\widetilde{\star})_{\tilde{\iota}}}=\widetilde{D}\right\} .
\end{aligned}
$$

Clearly $\mathcal{N}^{\widetilde{*}} \subseteq \overline{\mathcal{N}} \subseteq \widetilde{\mathcal{N}}$, in particular, $\bar{D}[X]_{\mathcal{N}^{\star}} \subseteq \bar{D}[X]_{\mathcal{N}} \subseteq \widetilde{D}[X]_{\widetilde{\mathcal{N}}}$. On the other hand, $\mathrm{Na}\left(\bar{D},(\widetilde{\star})_{\bar{\iota}}\right)=\bar{D}[X]_{\mathcal{N}}$ and $\mathrm{Na}\left(\widetilde{D},(\widetilde{\star})_{\widetilde{\iota}}\right)=\widetilde{D}[X]_{\widetilde{\mathcal{N}}}$. Recall that in the proof $\left(6_{\widetilde{\star}}\right) \Rightarrow\left(10_{\star_{f}}\right)$ of Theorem $\left[2.16\right.$, we have shown that $\bar{D}[X]_{\mathcal{N}^{\star}}=\widetilde{D}[X]_{\widetilde{\mathcal{N}}}$. Therefore, in particular, $\mathrm{Na}\left(\bar{D},(\widetilde{\star})_{\bar{\iota}}\right)=\mathrm{Na}\left(\widetilde{D},(\widetilde{\star})_{\widetilde{\iota}}\right)$. Henceforth, if $\left(6_{\widetilde{\star}}\right)$ holds then $\mathrm{Na}\left(\bar{D},(\widetilde{\star})_{\bar{\iota}}\right)\left(=\mathrm{Na}\left(\widetilde{D},(\widetilde{\star})_{\tilde{\iota}}\right)\right)$ is a Prüfer domain and so $\bar{D}$ is a $\mathrm{P}(\widetilde{\star})_{\bar{L}} \mathrm{MD}[20$, Theorem $3.1(\mathrm{i}) \Leftrightarrow(\mathrm{iii}))]$.

$\left(\overline{6}_{\widetilde{\star}}\right) \Rightarrow\left(6_{\widetilde{\star}}\right)$ By assumption and by $[20$, Theorem $\left.3.1(\mathrm{i}) \Leftrightarrow(\mathrm{iii}))\right] \mathrm{Na}\left(\bar{D},(\widetilde{\star})_{\bar{\iota}}\right)$ is a Prüfer domain and then obviously each overring of $\mathrm{Na}\left(\bar{D},(\widetilde{\star})_{\bar{\tau}}\right)$ is a Prüfer domain. In particular $\mathrm{Na}(\widetilde{D},(\widetilde{\star}) \widetilde{\iota})$ is a Prüfer domain and thus $\left(6_{\widetilde{\star}}\right)$ holds again by 20 , Theorem 3.1 (i) $\Leftrightarrow($ iii) $)$ ].

$\left(\overline{6}_{\widetilde{\star}}^{\prime}\right) \Leftrightarrow\left(\overline{6}_{\widetilde{\star}}\right)$ Note that, for each $E \in \overline{\boldsymbol{F}}(\bar{D})$, we have:

$$
E^{(\widetilde{\star}) \tau}=E^{\widetilde{\star}}=\bigcap\left\{E D_{P} \mid P \in \operatorname{QMax}^{\widetilde{\star}}(D)\right\}=(E \widetilde{D})^{(\widetilde{\star}) \tau}=E^{((\widetilde{\star}) \tilde{\iota})^{j}} .
$$

Therefore $(\widetilde{\star})_{\tau}=\left((\widetilde{\star})_{\widetilde{\imath}}\right)^{j}$. Henceforth it is straightforward that $\left(\overline{6}_{\widetilde{\star}}^{\prime}\right) \Rightarrow\left(\overline{6}_{\widetilde{\star}}\right)$ after recalling that $\left(\left(v_{\widetilde{D}}\right)^{j}\right)_{f}=\left(t_{\widetilde{D}}\right)^{j}$. Conversely, if $\left(\overline{6}_{\widetilde{\star}}\right)$ holds, we know already that 
$(\widetilde{\star})_{\widetilde{\iota}}=w_{\widetilde{D}}=t_{\widetilde{D}}$ (by the fact that $\left.\left(\overline{6}_{\widetilde{\star}}\right) \Rightarrow\left(6^{\prime} \widetilde{\star}\right)\right)$ and that $(\widetilde{\star})_{\tau}=\left((\widetilde{\star})_{\widetilde{\iota}}\right)^{j}=\left(t_{\widetilde{D}}\right)^{j}=$ $\left(\left(v_{\widetilde{D}}\right)^{j}\right)_{f}$.

(b) Let $D$ be a $\star_{f}$-quasi Prüfer domain. If $\bar{D}$ is $t$-linked to $(D, \star)$ then $\bar{D}$ is a $\mathrm{P} v_{\bar{D}} \mathrm{MD}$, since in this case $\bar{D}=\widetilde{D}$ (Lemma 2.9 and Theorem 2.16). On the other hand, if $\bar{D}$ is not $t$-linked to $(D, \star)$, then $\bar{D}$ is a $\mathrm{P}(\widetilde{\star})_{\tau} \mathrm{MD}$ (by (a)) and, since in this case $(\widetilde{\star})_{\bar{L}}$ is not a (semi)star operation on $\bar{D}$, we may not deduce that $\bar{D}$ is a $\mathrm{P} v_{\bar{D}} \mathrm{MD}$. However, in the previous Example 2.19, even if $\bar{D}$ is not $t$-linked to $\left(D, t_{D}\right)$, we do have that $\bar{D}$ is a $\mathrm{P} v_{\bar{D}} \mathrm{MD}$ because for $\bar{H} \in \operatorname{Max}^{t} \bar{D}(\bar{D})$ such that $\bar{H} \notin \operatorname{QMax}^{\left(w_{D}\right)_{\tau}}(\bar{D})$ we still have that $\bar{D}_{\bar{H}}$ is a valuation domain.

(c) Note that, if we replace $\star_{f}$ with $\star$ in the conditions $\left(4_{\star_{f}}\right),\left(8_{\star_{f}}\right),\left(9_{\star_{f}}\right)\left(10_{\star_{f}}\right)$ and $\left(14_{\star_{f}}\right)$ stated in Theorem 2.16, we obtain:

$\left(4_{\star}\right) D \subseteq K$ is a *-primitive extension.

$\left(8_{\star}\right)$ Every prime ideal of $\mathrm{Na}(D, \star)$ is extended from $D$.

$\left(9_{\star}\right) \mathrm{Na}(D, \star)$ is a quasi-Prüfer domain.

$\left(10_{\star}\right)$ The integral closure of $\mathrm{Na}(D, \star)$ is a Prüfer domain.

$\left(14_{\star}\right) D$ is a $t$-quasi-Prüfer domain (or a UMt-domain) and $\widetilde{\star}=w$.

It is trivial from the definitions that the previous conditions coincide with the analogous conditions stated for $\star_{f}$ in Theorem 2.16.

A natural question arises from this observation: is it possible to find suitable characterizations of the $\star$-quasi-Prüfer domains, by "weakening" the remaining conditions in Theorem 2.16?

(d) Recall that Houston and Zafrullah have recently introduced the UMv-domains, i.e., the integral domains $D$, such that each upper to zero is a maximal $v_{D[X]}$-ideal of $D[X]$. It is known that $\mathrm{UM} v$-domains are characterized by the fact that, for each upper to zero $P, \boldsymbol{c}_{D}(P)^{v_{D}}=D$ and $((P: P)=) D[X] \subsetneq P^{-1}$ [43, Theorem 2.2]. On the other hand, if $D$ is a $\mathrm{UM} v$-domain and if $P$ is a $v_{D}$-prime ideal of $D$, then the integral closure of $D_{P}$ is a Prüfer domain [43, Theorem 3.6], i.e., $D_{P}$ is a quasi-Prüfer domain by Theorem $1.1((1) \Leftrightarrow(6))$. Therefore, by Lemma 2.1 $((\mathrm{iv}) \Rightarrow(\mathrm{i}))$, a $\mathrm{UM} v$-domain is a $v$-quasi-Prüfer domain.

Note also that a UM $v$-domain is not necessarily a $t$-quasi-Prüfer domain (= $\mathrm{UM} t$-domain). To see this, let $D$ be a $v$-domain (i.e., an integral domain such that each nonzero finitely generated ideal is $v$-invertible [27, Theorem 34.6]) which is not a PvMD (cf. [27, Exercise 5, page 425] and also [8, §3], [36] and [37]). A ring of this type must admit an upper to zero which is a maximal $v$-ideal but not a maximal $t$-ideal, since it is an integrally closed $\mathrm{UM} v$ domain which is not a $\mathrm{UM} t$-domain (Remark 1.2 (d) and [43, Theorem $3.3((1) \Leftrightarrow(2))]$ ). This example also shows that a $v$-quasi-Prüfer domain need not be a $v_{f}$-quasi-Prüfer domain (cf. Example 2.2).

Question: Is it possible to find a $v$-quasi-Prüfer domain which is not a $\mathrm{UM} v$ domain?

(e) Houston and Zafrullah [43, Proposition 4.6] proved that $D$ is a UMt-domain if and only if each upper to zero of the form $(a X+b) K[X] \cap D[X]$, where $0 \neq a, b \in$ $D$, is a maximal $t$-ideal of $D[X]$ or, equivalently, each upper to zero of the form $(a X+b) K[X] \cap D[X]$, where $0 \neq a, b \in D$, contains a nonzero polynomial $g$ with $\boldsymbol{c}_{D}(g)^{t}=D$ [42, Theorem 1.4].

A similar characterization holds for $\star_{f}$-quasi-Prüfer domains. More precisely, given a semistar operation $\star$ on an integral domain $D$, the following are equivalent:

$\left(1_{\star_{f}}\right) D$ is a $\star_{f}$-quasi-Prüfer domain. 
$\left(2_{\star_{f}}^{\prime}\right)$ Each upper to zero in $D[X]$ of the form $(a X+b) K[X] \cap D[X]$ contains a nonzero polynomial $g$ with $\boldsymbol{c}_{D}(g)^{\star}=D^{\star}$.

$\left(2_{\star_{f}}^{\prime \prime}\right)$ For each nonzero $h \in D[X]$, there exists $0 \neq g \in h K[X] \cap D[X]$ with $\boldsymbol{c}_{D}(g)^{\star}=D^{\star}$.

$\left(1_{\star_{f}}\right) \Leftrightarrow\left(2_{\star_{f}}^{\prime}\right)$ By using the equivalence $\left(1_{\star_{f}}\right) \Leftrightarrow\left(4_{\star_{f}}\right)$ of Theorem 2.16 and the previous point (a), it is enough to show that $Q:=(a X+b) K[X] \cap D[X]$ contains a nonzero polynomial $g$ with $\boldsymbol{c}_{D}(g)^{\star}=D^{\star}$ if and only if $u=-\frac{b}{a}$ is $\star$-primitive over D.

For the "only if" part, let $0 \neq g \in Q$ such that $\boldsymbol{c}_{D}(g)^{\star}=D^{\star}$. Clearly $g=(a X+$ $b) h$, for some $h \in K[X]$. Then $g(u)=\left(a\left(-\frac{b}{a}\right)+b\right) h(u)=0$, thus $u$ is $\star$-primitive over $D$. For the "if" part, suppose that $u\left(=-\frac{b}{a}\right)$ is $\star$-primitive over $D$. Then there exists a nonzero polynomial $g \in D[X]$ such that $\boldsymbol{c}_{D}(g)^{\star}=D$ and $g(u)=0$. Therefore in $K[X]$ we have $g=(a X+b) h+r$, where $h \in K[X]$ and $r$ is a constant in $K$. Since $g(u)=0$, we have $r=0$, and thus $g \in Q=(a X+b) K[X] \cap D[X]$.

The implication $\left(2_{\star_{f}}^{\prime \prime}\right) \Rightarrow\left(2_{\star_{f}}\right)$ is obvious.

$\left(2_{\star_{f}}\right) \Rightarrow\left(2_{\star_{f}}^{\prime \prime}\right)$ Let $h K[X]=\ell_{1} \ell_{2} \ldots \ell_{n} K[X]$, where $\ell_{i} \in D[X]$ is irreducible in $K[X]$, for $1 \leq i \leq n$. Since $Q_{i}:=\ell_{i} K[X] \cap D[X]$ is an upper to zero, then we can find $0 \neq g_{i} \in Q_{i}$ such that $\boldsymbol{c}_{D}\left(g_{i}\right)^{\star}=D^{\star}$. Then $g:=g_{1} g_{2} \ldots g_{n} \in h K[X] \cap D[X]$ and it is not difficult to see that $\boldsymbol{c}_{D}(g)^{\star}=D^{\star}$.

(f) Note that, from the equivalence $\left(1_{\star_{f}}\right) \Leftrightarrow\left(6_{\widetilde{\star}}\right)$ in Theorem 2.16 (or, from Corollary 2.17), we deduce that if $\star$ is a (semi)star operation on $D$, then $D$ is an integrally closed $\star_{f}$-quasi-Prüfer domain if and only if $D$ is a $\mathrm{P} \approx \mathrm{MD}$ (or, equivalently, a $\mathrm{P} \star \mathrm{MD})$. This result generalizes the statement on $\mathrm{P} v$ MDs recalled in Remark 1.2 (d).

Corollary 2.21. With the notation of Theorem 2.16, we have that $\left(1_{\star_{f}}\right)$ is equivalent to

$\left(12_{\star_{f}}^{\prime}\right)$ Each $t$-linked overring $R$ to $\left(D, \star_{f}\right)$ is a $t_{R}$-quasi-Prüfer domain and each $(\widetilde{\star})_{\iota}$-maximal ideal of $R$ is a $t_{R}$-ideal, where $\iota: D \hookrightarrow R$ is the canonical embedding.

Proof. $\left(1_{\star_{f}}\right) \Rightarrow\left(12_{\star_{f}}^{\prime}\right)$ Note that from the proof $\left(1_{\star_{f}}\right) \Rightarrow\left(12_{\star_{f}}\right)$ of the previous Theorem 2.16, we deduce, without assuming that $\star$ is a (semi)star operation on $D$, that $R$ is a $(\widetilde{\star})_{\iota}$-quasi-Prüfer domain. Henceforth $R$ is also a $t_{R}$-quasi-Prüfer domain since $(\widetilde{\star})_{\iota}$ is a (semi)star operation of finite type on $R$. Now applying the implication $\left(1_{\star_{f}}\right) \Rightarrow\left(12_{\star_{f}}\right)$ to $R$ and to the (semi)star operation $(\widetilde{\star})_{\iota}$, since $R$ is trivially $t$-linked to $\left(R,(\widetilde{\star})_{\iota}\right)$, we have in particular that each $(\widetilde{\star})_{\iota}$-maximal ideal of $R$ is a $t_{R^{-} \text {-ideal. }}$

$\left(12_{\star_{f}}^{\prime}\right) \Rightarrow\left(11_{\star_{f}}\right)$ If $P \in \operatorname{QMax}^{\star_{f}}(D)=\operatorname{QMax}^{\widetilde{\star}}(D)$, then clearly $\left(D_{P}\right)^{\widetilde{\star}}=$ $\bigcap\left\{D_{P} D_{M} \mid M \in \operatorname{QMax}^{\star_{f}}(D)\right\}=D_{P}$ and so $D_{P}$ is $t$-linked to $\left(D, \star_{f}\right)$ (Lemma 2.9 $((\mathrm{v}) \Rightarrow(\mathrm{i})))$. Therefore, by assumption, $D_{P}$ is a $t_{D_{P} \text {-quasi-Prüfer domain. More- }}$ over, clearly $P D_{P}$ is a maximal $(\widetilde{\star})_{\iota}$-ideal of $D_{P}$ and so it is a $t_{D_{P}}$-ideal of $D_{P}$. Then $D_{P}$ is a quasi-Prüfer domain by Corollary $1.3((13) \Rightarrow(1))$.

Corollary 2.22. If $D$ is a $\star_{f}$-quasi-Prüfer domain, then

(a) If $P$ is a nonzero prime ideal of $D$ and if $P^{\star_{f}} \neq D^{\star}$ (e.g., if $P$ is a quasi$\star_{f}$-prime ideal of $\left.D\right)$, then $P=P^{\star_{f}}=P^{t}$. 
(b) $\operatorname{dim}^{\widetilde{\star}}(D)=\operatorname{dim}_{f}^{{ }_{f}}(D)=\operatorname{dim}^{t}(D)=\operatorname{dim}^{t}(\mathrm{Na}(D, \star))=\operatorname{dim}(\mathrm{Na}(D, \star))$.

Proof. (a) It suffices to show that $P$ is a $t$-ideal. Let $Q$ be a quasi- $\star_{f}$-maximal ideal of $D$ containing $P$. Then $D_{Q}$ is a quasi-Prüfer domain (Theorem $2.16\left(\left(1_{\star_{f}}\right) \Rightarrow\right.$ $\left.\left(11_{\star_{f}}\right)\right)$ ), and since $P D_{Q}$ is a proper prime ideal of $D_{Q}, P D_{Q}$ is a prime $t$-ideal in $D_{Q}$ (Corollary [1.3), and hence $P=P D_{Q} \cap D$, is a $t$-ideal of $D$ [46, Lemma 3.17 (1)].

(b) Note that $\operatorname{dim}^{\star_{f}}(D)=\operatorname{dim}^{t}(D)$ by (1) and $\operatorname{dim}^{t}(\mathrm{Na}(D, \star))=\operatorname{dim}(\mathrm{Na}(D, \star))$ by Corollary 1.3 and Theorem $2.16\left(\left(1_{\star_{f}}\right) \Rightarrow\left(9_{\star_{f}}\right)\right)$. Recall that $M \in \operatorname{Max}(\operatorname{Na}(D, \star))$ if and only if $M \cap D \in \operatorname{QMax}^{\star_{f}}(D)$ [24, Proposition 3.1 (5)]. Since each prime ideal of $\mathrm{Na}(D, \star)$ is extended from $D$ (Theorem $2.16\left(\left(1_{\star_{f}}\right) \Rightarrow\left(8_{\star_{f}}\right)\right)$ ), we have $\operatorname{dim}^{\star}(D)=\operatorname{dim}(\operatorname{Na}(D, \star))$. The first equality follows from the fact that the notions of $\star_{f}$-quasi-Prüfer domain and $\widetilde{\star}$-quasi-Prüfer domain coincide (Corollary 2.4 (c)) and from the fact that $\mathrm{Na}(D, \widetilde{\star})=\mathrm{Na}\left(D, \star_{f}\right)=\mathrm{Na}(D, \star)$.

It is well known that if $M$ is a maximal $t$-ideal of $D[X]$, then either $M \cap D=(0)$ or $M=(M \cap D)[X]$ [2, Proposition 1.1] and $I$ is a $t$-ideal of $D$ if and only if $I[X]$ is a $t$-ideal of $D[X]$ [46, Corollary 2.3]. Thus $\left.\operatorname{dim}^{t}(D) \leq \operatorname{dim}^{t}(D[X]) \leq 2 \operatorname{dim}^{t}(D)\right)$ (cf. also [38, page 169] and [57, Section 3]).

Recall that Kang proved that if $D$ is a $\mathrm{P} v \mathrm{MD}$ then $\operatorname{dim}^{t}(D)=\operatorname{dim}(\mathrm{Na}(D, v))$ 46, Theorem 3.22]. The following corollary extends Kang's result to the UMt-domains.

Corollary 2.23. Let $D$ be a UMt-domain which is not a field and let $X$ be an indeterminate over $D$. Then $\operatorname{dim}^{w}(D)=\operatorname{dim}^{t}(D)=\operatorname{dim}^{t}(D[X])=\operatorname{dim}^{t}(\mathrm{Na}(D, v))=$ $\operatorname{dim}(\mathrm{Na}(D, v))$.

Proof. As we already remarked in general $\operatorname{dim}^{t}(D) \leq \operatorname{dim}^{t}(D[X])$. Let $Q$ be a maximal $t$-ideal of $D[X]$. If $Q \cap D=(0)$, then obviously ht $(Q)=1 \leq \operatorname{dim}(\mathrm{Na}(D, v))$. If $Q \cap D \neq(0)$, then $Q=(Q \cap D)[X]$ and hence $Q \cap \mathcal{N}^{v}=\emptyset$. Therefore $Q N a(D, v) \neq$ $\mathrm{Na}(D, v)$ and so ht $(Q) \leq \operatorname{dim}(\mathrm{Na}(D, v))$, hence $\operatorname{dim}^{t}(D[X]) \leq \operatorname{dim}(\mathrm{Na}(D, v))$. The conclusion follows easily from Corollary 2.22 (b).

Remark 2.24. (a) Note that, for a UMt-domain, Wang [58, Theorem 2.6] proved already the equality $\operatorname{dim}^{w}(D)=\operatorname{dim}(\mathrm{Na}(D, v))$.

(b) It is clear that, in general, $\operatorname{dim}^{t}(D) \leq \operatorname{dim}^{w}(D)$, since each $t$-ideal is also a $w$-ideal and it is easy to see that (in the non UMt-domain case) it can happen that $\operatorname{dim}^{t}(D) \neq \operatorname{dim}^{w}(D)$. For instance, let $R$ be a quasi-local factorial domain of dimension 3 with maximal ideal $M$. Set $F:=R / M$ and let $\varphi: R \rightarrow F$ be the canonical homomorphism. Assume that $k$ is a proper subfield of $F$, set $D:=\varphi^{-1}(k)$ and let $Q$ is a prime ideal of $D$ and $R$ such that ht $(Q)=2$. Clearly, since $R$ is a UFD and $M=(D: R)$, then $M=M^{v_{D}}=M^{w_{D}} \subsetneq M^{w_{R}}=M^{t_{R}}=M^{v_{R}}=R$ and $Q=Q^{w_{D}} \subsetneq Q^{t_{R}}=Q^{v_{R}}=R$ (note that $Q=Q^{w_{D}}$, since $\operatorname{Max}^{w_{D}}(D)=\operatorname{Max}(D)$ and so $w_{D}$ coincides with the identity (semi)star operation on $D$ ). Let $I \subseteq Q$ be a nonzero finitely generated ideal of $D$ with $(R:(R: I))=(R:(R: I R))=R$ or, equivalently, $(R: I)=R$. Hence $(D: I) \subseteq(R: I)=R=(M: M)=(D:$ $M) \subseteq(D: I)$ and so $(D: I)=R$. Therefore $I^{v_{D}}=(D:(D: I))=(D: R)=M$ and so $M=I^{v_{D}} \subseteq Q^{t_{D}} \subseteq M^{t_{D}}=M$. Henceforth $Q^{t_{D}}=M$. Therefore we have $\operatorname{dim}^{t}(D)=2 \lessgtr \operatorname{dim}(D)=\operatorname{dim}^{w}(D)=3$.

(c) It is well known that an integral domain $D$ is Prüfer domain (resp., $\mathrm{P} v \mathrm{MD}$ ), if and only if each nonzero two generated ideal of $D$ is invertible (resp., $t$-invertible) 
27. Theorem 22.1] (resp., [48, Lemma 1.7]). In case $*$ is a star operation of finite type, it is known that $D$ is $\mathrm{P} * \mathrm{MD}$ if and only if each (nonzero) two generated ideal of $D$ is $*$-invertible [40, Theorem 1.1]. It is natural to ask whether a similar result holds in the semistar setting. Let $\star$ be a semistar operation on an integral domain $D$. Recall that, in [25. Theorem 2.3], it is shown that for $I \in \boldsymbol{f}(D), I$ is $\star_{f}$-invertible if and only if $I D_{Q}$ is principal, for each $P \in \operatorname{QMax}^{\star_{f}}(D)$. Moreover, it is well known that, for a local domain, the following properties are equivalent [27, Theorem 22.1]:

(i) Every nonzero finitely generated ideal is principal;

(ii) Every two generated is principal;

(iii) $R$ is a valuation domain.

On the other hand, $D$ is a $\mathrm{P} \star \mathrm{MD}$ if and only if $D_{P}$ is a valuation domain, for each $P \in \operatorname{QMax}^{\star_{f}}(D)$ [20, Theorem 3.1]. Therefore, by the previous considerations it follows that $D$ is a $P \star M D$ if and only if each (nonzero) two generated ideal of $D$ is $\star_{f}$-invertible.

\section{ACKNOWLEDGMENTS}

During the preparation of this paper, the second named author was partially supported by a grant PRIN-MiUR.

\section{REFERENCES}

[1] M.F. Atiyah and I.G. Macdonald, Introduction to Commutative Algebra, AddisonWesley, Reading, Massachusetts, 1969.

[2] D.D. Anderson, Star-operations induced by overrings, Comm. Algebra 16(1988), 2535-2553.

[3] D.F. Anderson, D.E. Dobbs, and M. Fontana, On treed Nagata rings, J. Pure Appl. Algebra 61(1989), 107-122.

[4] V. Barucci and S. Gabelli, How far is a Mori domain from being a Krull domain?, J. Pure Appl. Algebra, 45(1987), 101-112.

[5] A. Ayache, P.-J. Cahen, and O. Echi, Anneaux quasi-Prüfériens et P-anneaux, Boll. Un. Mat. Ital 10-B (1996), 1-24.

[6] G.W. Chang, *-Noetherian domains and the ring $D[X]_{N_{*}}$, J. Algebra 297(2006), 216-233.

[7] G.W. Chang and M. Zafrullah, The w-integral closure of integral domains, J. Algebra 259(2006), 195-210.

[8] J. Dieudonné, Sur la théorie de la divisibilité, Bull. Soc. Math. France 69(1941), 133-144.

[9] A. De Souza Doering and Y. Lequain, Chain of prime ideals in polynomial rings, J. Algebra 78(1982), 163-180.

[10] D.E. Dobbs, On INC-extensions and polynomials with unit content, Canad. Math. Bull. 23(1980), 37-42.

[11] D.E. Dobbs, E.G. Houston, T.G. Lucas, and M. Zafrullah, t-linked overrings as intersections of localizations, Proc. Amer. Math. Soc. 109(1990), 637-646.

[12] D.E. Dobbs, E.G. Houston, T.G. Lucas, and M. Zafrullah, t-linked overrings and Prüfer $v$-multiplication domains, Comm. Algebra 17(1989), 2835-2852.

[13] D.E. Dobbs, E.G. Houston, T.G. Lucas, M. Roitman, and M. Zafrullah, On t-linked overrings, Comm. Algebra 20(1992), 1463-1488.

[14] T. Dumitrescu, A two-dimensional domain whose integral closure is not t-linked, An. St. Univ. Ovidius Constanţa 9(2001), 55-58.

[15] S. El Baghdadi and M. Fontana, Semistar linkedness and flatness, Prüfer semistar multiplication domains, Comm. Algebra 32 (2004), 1101-1126.

[16] S. El Baghdadi, M. Fontana, and G. Picozza, Semistar Dedekind domains, J. Pure Appl. Algebra 193 (2004), 27-60.

[17] M. Fontana, Topologically defined classes of commutative rings, 123(1980), 331-355.

[18] M. Fontana and J.A. Huckaba, Localizing systems and semistar operations, "Non-Noetherian Commutative Ring Theory" (S. T. Chapman and S. Glaz, eds.), Kluwer Academic Publishers, 2000, pp. 169-198. 
[19] M. Fontana, J. Huckaba, and I. Papick, Prüfer domains, Marcel Dekker, 1997.

[20] M. Fontana, P. Jara, and E. Santos, Prüfer $\star$-multiplication domains and semistar operations, J. Algebra Appl. 2 (2003), 21-50.

[21] M. Fontana, S. Gabelli, and E. Houston, UMT-domains and domains with Prüfer integral closure, Comm. Algebra 26(1998), 1017-1039.

[22] M. Fontana and K. A. Loper, A Krull-type theorem for the semistar integral closure of an integral domain, ASJE Theme Issue "Commutative Algebra" 26(2001), 89-95.

[23] M. Fontana and K.A. Loper, Kronecker function rings: a general approach, in Ideal Theoretic Methods in Commutative Algebra, Lecture Notes in Pure Appl. Math., Marcel Dekker, 220(2001), 189-205.

[24] M. Fontana and K.A. Loper, Nagata rings, Kronecker function rings and related semistar operations, Comm. Algebra 31(2003), 4775-4801.

[25] M. Fontana and G. Picozza, Semistar invertibility on integral domains, Algebra Colloq. $12(2005), 645-664$.

[26] R. M. Fossum, The divisor class group of a Krull domain, Springer 1973.

[27] R. Gilmer, Multiplicative Ideal Theory, Marcel Dekker, New York, 1972.

[28] R. Gilmer and J. F. Hoffmann, A characterization of Prüfer domains in terms of polynomials, Pacific J. Math., 60(1975), 81-85.

[29] S. Glaz and W.V. Vasconcelos, Flat ideals, II, Manuscripta Math. 22(1977), 325-341.

[30] M. Griffin, Some results on v-multiplication rings, Canad. J. Math 19(1967), 710-722.

[31] F. Halter-Koch, Generalized integral closures, in "Factorization in Integral Domains" (D.D. Anderson, Editor), Lecture Notes Pure Appl. Math., Marcel Dekker, 187(1997), 349-358.

[32] F. Halter-Koch, Ideal Systems: An Introduction to Multiplicative Ideal Theory, Marcel Dekker, New York, 1998.

[33] J.R. Hedstrom and E.G. Houston, Pseudo-valuation domains, Pacific J. Math. 75(1978), 137-147.

[34] J. R. Hedstrom and E. G. Houston, Pseudo-valuation domains II, Houston J. Math. 4(1978),199-207.

[35] W. Heinzer, J. Ohm, and R.L. Pendleton, On integral domains of the form $\cap D_{P}, P$ minimal, J. Reine Angew Math. 241(1970), 150-159.

[36] W. Heinzer, An essential integral domain with a nonessential localization, Canad. J. Math. 23(1981), 400-403.

[37] W. Heinzer and J. Ohm, An essential ring which is not a v-multiplication ring, Canad. J. Math. 21(1972), 856-861.

[38] E. Houston, Prime t-ideals in $R[X]$, in "Commutative Ring Theory", P.-J. Cahen, D.G. Costa, M. Fontana, S.-E. Kabbaj (Eds.), Lecture Notes Pure Appl. Math., Marcel Dekker, 153(1994), 163-170.

[39] E. Houston, Uppers to zero in polynomial rings, in "Multiplicative Ideal Theory in Commutative Algebra. A Tribute to the Work of Robert Gilmer", Brewer, J.W., Glaz, S., Heinzer, W.J., Olberding, B.M. (Eds.), Springer, 2006, pp. 243-261.

[40] E. Houston, S. Malik, and J. Mott, Characterizations of *-multiplication domains, Canad. Math. Bull. 27(1984), 48-52.

[41] E. Houston and J. Taylor, Arithmetic properties in pullbacks, J. Algebra 310(2007), 235-260.

[42] E. Houston and M. Zafrullah, On t-invertibility, II, Comm. Algebra 17(1989), 1955-1969.

[43] E. Houston and M. Zafrullah, UMV-domains, in "Arithmetical Properties of Commutative Rings and Monoids", Lecture Notes Pure Appl. Math., Chapman and Hall, 241(2005), 304315.

[44] J. Huckaba, Commutative rings with zero divisors, Marcel Dekker, 1988.

[45] P. Jaffard, Les Systèmes d'Idéaux, Dunod, Paris, 1960.

[46] B.G. Kang, Prüfer v-multiplication domains and the ring $R[X]_{N_{v}}$, J. Algebra 123(1989), 151-170.

[47] I. Kaplansky, Commutative rings, Revised Ed., University of Chicago, Chicago, 1974.

[48] S. Malik, J.L. Mott, and M. Zafrullah, On t-invertibility, Comm. Algebra 16(1988), 149-170.

[49] A.Mimouni, Integral domains in which each ideal is a w-ideal, Comm. Algebra 33(2005), $1345-1355$.

[50] J.L. Mott, and M. Zafrullah, On Prüfer v-multiplication domains, Manuscripta Math. 35 (1981), 1-26. 
[51] A. Okabe and R. Matsuda, Star operations and generalized integral closures, Bull. Fac. Sci., Ibaraki Univ. 24(1992), 7-13.

[52] A. Okabe and R. Matsuda, Semistar operations on integral domains, Math. J. Toyama Univ. 17(1994), 1-21.

[53] M. Nagata, Local rings, New York, Interscience, 1962.

[54] I.J. Papick, Super-primitive elements, Pacific J. Math. 105(1983), 217-226.

[55] G. Picozza, Semistar operations and multiplicative ideal theory, Ph.D. Thesis, Università degli Studi "Roma Tre", 2004.

[56] G. Picozza and F. Tartarone, When the semistar operation $\widetilde{\star}$ is the identity, Comm. Algebra (to appear).

[57] F.G. Wang, On t-dimension of polynomial rings, Preprint (Sichuan Normal University, Chendu, 2005).

[58] F.G. Wang, w-dimension of a domain, Comm. Algebra 27(1999), 2267-2276.

[59] F.G. Wang, On induced operations and UMT-domains, Sichuan Shifan Daxue Xuebao Ziran Kexue Ban 27(2004), 1-9.

[60] F.G. Wang and R.L. MacCasland, On w-modules over strong Mori domains, Comm. Algebra 25(1997), 1285-1306.

[61] F.G. Wang and R.L. MacCasland, On strong Mori domains, J. Pure Appl. Algebra 135(1999), 155-165.

[62] M. Zafrullah, Putting t-invertibility to use, "Non-Noetherian Commutative Ring Theory", S. T. Chapman and S. Glaz (Eds.), Kluwer Academic Publishers, 2000, pp. 429-457. 